\title{
Quasicontinuum simulation of fracture at the atomic scale
}

\author{
R Miller†, E B Tadmor†, R Phillipsł and M Ortiz§ \\ $\dagger$ Division of Engineering and Applied Science, Harvard University, Cambridge, MA 02138, \\ USA \\ $\ddagger$ Division of Engineering, Brown University, Providence, RI 02912, USA \\ $\S$ Department of Aeronautics, California Institute of Technology, Pasadena, CA 91125, USA
}

Received 23 March 1998, accepted for publication 14 June 1998

\begin{abstract}
We study the problem of atomic scale fracture using the recently developed quasicontinuum method in which there is a systematic thinning of the atomic-level degrees of freedom in regions where they are not needed. Fracture is considered in two distinct settings. First, a study is made of cracks in single crystals, and second, we consider a crack advancing towards a grain boundary (GB) in its path. In the investigation of single crystal fracture, we evaluate the competition between simple cleavage and crack-tip dislocation emission. In addition, we examine the ability of analytic models to correctly predict fracture behaviour, and find that the existing analytical treatments are too restrictive in their treatment of nonlinearity near the crack tip. In the study of GB-crack interactions, we have found a number of interesting deformation mechanisms which attend the advance of the crack. These include the migration of the GB, the emission of dislocations from the GB, and deflection of the crack front along the GB itself. In each case, these mechanisms are rationalized on the basis of continuum mechanics arguments.
\end{abstract}

\section{Introduction}

The mechanisms that control fracture in materials are complex and diverse, and span length scales from the angstrom to the millimetre. At the most fundamental level, fracture is simply the breaking of atomic bonds at the advancing crack front. However, the interactions between such a crack and the myriad of possible material microstructures and defects imply that fracture mechanics in general may admit of no simple unifying description. Consider, for example, even the simplest case of a crack advancing through a homogeneous, amorphous solid. Even this example is not simple to understand, as dynamic instabilities lead to different behaviour at different stages of crack propagation (Sharon et al 1996). This is seen in the so-called 'mirror-mist-hackle' fracture regimes (Lawn 1993). In heterogeneous materials, a great many mechanisms may conspire to produce the macroscopically observed fracture behaviour. Steels, in which there are hard second-phase particles, undergo fracture by a complicated interaction between dislocation motion and multiplication, void formation and growth, and cleavage on atomic planes. In ceramic polycrystals such as alumina, the competition between crack propagation through the crystalline grains or along the grain boundaries (GBs) can profoundly influence the toughness of the bulk material (Lawn 1993). Composite materials such as fibreglass and wood illustrate a still larger set of length scales that participate in fracture processes, as the bonding between the reinforcing structures and matrix become important. Indeed, there are a great many considerations in the modelling of fracture processes. In this work, we focus on the elementary mechanisms associated with the specific example of fracture in single and polycrystalline metals. 
In polycrystalline and multiphase materials, the interactions between the advancing crack, GBs, and dislocations are a central part of the fracture process, and correctly describing the interactions between these constituents presents a unique challenge to any modelling effort. On the one hand, atomic scale information is essential to this description, as the energetics of dislocations and GBs are ultimately controlled by atomic interactions. On the other hand, the modelling of systems with macroscopic dimensions and boundary conditions is not possible within a fully atomistic framework. In this paper, we study fracture at the atomic scale using the recently developed quasicontinuum (QC) method of Tadmor et al (1996), a mixed atomistic and continuum approach. Specifically, we consider fracture in single crystals of nickel, and then consider the interactions between cracks and GBs in nickel bicrystals.

Until recently, most of the effort in fracture mechanics has focused on phenomenological descriptions of material behaviour (see Freund (1990) for a review). In this setting, dissipative deformation processes around a crack tip are treated using continuum plasticity models, while fracture criteria are inserted into the model based either on the Griffith analysis or via a Barenblatt type of model. While these continuum models have been very successful in describing the fracture of materials, they are incapable of elucidating the precise deformation mechanisms near the advancing crack tip. The perspective adopted here is that through the inclusion of atomic scale information it is possible to understand the mechanisms that ultimately conspire to produce the phenomena mimicked in the continuum mechanics approach.

The importance of atomic scale effects in fracture has been recognized for some time, and work to understand atomic scale fracture initially focused on analytic models that included lattice discreteness in an approximate way (Thomson 1973). Early computer simulations of fracture (see, for example, Sinclair and Lawn (1972)) were limited by the available computational power, but led to significant insight into atomic configurations at the crack tip and the effects of lattice trapping. Lately, advances in atomistic simulation of materials and increases in computational resources have made it possible to simulate fracture processes at the atomic scale for relatively large systems. Approaches to modelling fracture at the atomic scale range from the conceptually simple but computationally challenging fully atomistic approach, to a variety of mixed atomistic/continuum models that include atomistic effects either through some approximate scheme or by embedding an atomistic crack-tip region in a bulk continuum.

The first approach, that of full molecular dynamics or lattice statics simulations of fracture, has been pursued by a number of groups (Zhou et al 1997, Abraham et al 1998, Paskin et al 1985, Lomdahl et al 1993, Holian and Ravelo 1995, Daw et al 1986, Cleri et al 1998b, Cheung and Yip 1990). Atomistic simulations of fracture are carried out by building a small crystal with an attendant crack tip and applying a strain rate to the crystal boundaries. The number of atoms in such a simulation has steadily been increasing as computer power allows, from a few thousand atoms in the earlier works to tens of millions of atoms most recently. The challenges in such an approach come both from the huge computational power and storage required to explicitly model millions of atoms, as well as from the need to make sense of the vast quantities of information which result. Simulations of $10^{8}$ atoms are now fairly routine, but such calculations require the resources of massively parallel supercomputers. While such calculations are an impressive demonstration of advances in computation, the task of extracting an understanding of material behaviour which can be used as a basis for higher-level models remains daunting.

Despite the challenges of explicitly treating millions of atomic degrees of freedom, fully atomistic studies of fracture have played an important role in explicating the atomic- 
level mechanisms of fracture. First, molecular dynamics is a fertile setting within which to document and classify the atomic-level mechanisms of fracture. They also provide a highly controlled 'experiment' with which to test the validity of analytic models. In some cases, such as in the work of Paskin et al (1985) and Cleri et al (1998b), atomistic simulation has been used to determine the point at which continuum assumptions fail. In other cases, the simulations have shed light on counter-intuitive results that may not have been otherwise revealed. For example, Abraham et al (1998) showed that the cleavage planes in facecentred cubic (fcc) crystals cannot be determined simply from a rank ordering of surface energies.

Perhaps the most frustrating aspect of such large-scale atomistic calculations is that only a small subset of the atomic degrees of freedom do anything especially interesting. A great many of the atoms respond in a way that could be described by elasticity theory, and yet the storage and book-keeping associated with their degrees of freedom-necessary if a purely atomistic approach is used-consumes a large proportion of the computational time. The motivation for a number of researchers has been this realization that despite the need for full atomic detail near the crack tip, the majority of atoms behave in an elastic fashion. As a result, there are now several modelling approaches which aim to reduce the number of degrees of freedom in atomic scale systems. Some of these models, like that of Kohlhoff et al (1991) and Gumbsch (1995), effect this reduction by dividing the problem into two distinct zones, one which is fully atomistic and the other which is modelled using conventional finite elements. Another example is the approach of Zhou et al (1993, 1994) and Schiotz et al (1997). In this instance, the actual number of atomic degrees of freedom is not changed, but rather a large proportion of the atoms are treated using a linear approximation to their response. These linear atoms, while they are still explicitly modelled, demand considerably less computational overhead than fully nonlinear atoms, and hence the approach greatly reduces the calculation time. A third method, and that which will be used here, is the QC method. In this approach, the machinery of finite elements is combined with an atomistic constitutive model to significantly reduce the number of atoms whose degrees of freedom must be explicitly considered. One of the advantages of the QC approach is that it is adaptive, meaning that the subset of the atomic degrees of freedom which are explicitly included in the model can change in response to an evolving deformation. In this way, the initial design of the model does not restrict the deformation of the body to predetermined pathways. Currently, the QC method is limited to static energy minimization, and thus fracture calculations must be carried out in a quasistatic framework. In this way, the QC method is an alternative to lattice statics calculations of fracture processes.

These mixed approaches can be used to investigate fracture in much the same way as full atomistics, with the advantage of reduced computational demand. For example, Gumbsch (1995) studied brittle cracks in fcc crystals under mixed loading, and was able to compute critical loads to failure for sharp cracks and for cracks with atomic scale blunting at the tip. Zhou et al $(1993,1994)$ used their simulations to test analytic models of fracture. They determined that the analytic models were ignoring an important contribution to the energetics of crack-tip deformation-namely, the energy of the ledge which forms when a crack tip emits a dislocation. Finally, Schiotz et al (1997) showed how atomic scale crack blunting can lead to increased resistance to cleavage along atomic planes and possibly enhanced ductility.

The mixed atomistic/continuum strategies just outlined, like full atomistics, still suffer from a heavy reliance on numerical simulation. More to the point, analytical progress within such models is difficult. Continuum-based models of fracture which include discrete lattice effects in a minimalistic way have been proposed which have lead to important 
insights and the ability to make predictions about how the atomic scale will effect the fracture behaviour of materials. The most commonly advanced viewpoint in such models is that crack-tip deformation is a competition between cleavage of atomic planes and the emission of dislocations from the crack tip (see, for example, Armstrong (1966), Kelly et al (1967), and Rice and Thomson (1974)). A recent attempt to model this competition between dislocation emission and brittle cleavage is the model of Rice (1992), where the effects of the discrete lattice are included by introducing a single slip plane at the tip of the crack and treating slip on that plane via a Peierls-Nabarro dislocation model. This model has generated considerable interest due to its tractable formulation and ability to make quantitative predictions about material behaviour. The Rice framework has also been extended to a fully three-dimensional (3D) model by Xu et al (1995), revealing the clear need for proper treatment of the third dimension in fracture modelling. A number of authors have studied the agreement between the Rice model and atomistic simulation, and have subsequently sought refinements to the model (Thomson and Carlsson 1994, Juan and Kaxiras 1996, Gumbsch and Beltz 1995, Sun et al 1993, Zhou et al 1994). Thus, an important role for numerical studies of atomic scale fracture is to assess the accuracy of these continuum models.

In all of the models discussed to this point, the focus has been on a crack in an otherwise perfect single crystal. While understanding that this simplified problem is clearly important to the larger story of material fracture, it does not begin to probe the complex interactions between cracks and microstructure so often observed in experiment (Lawn 1993, Wang and Anderson 1991). The presence of GBs in a sample immediately presents additional mechanisms for permanent deformation, which are absent from a single crystal. These include, for instance, the migration of the GBs themselves (Babcock and Balluffi 1989, Würschum and Balluffi 1993), the possibility for GBs to act as sources for new dislocations (Li 1981), and the transmission of dislocations between grains (Shen et al 1988). It is hoped that through atomistic simulation, specific questions about these mechanisms may be answered. For instance, atomistic calculation may lead to a better understanding of the kinetics of GB migration, or of the conditions under which slip transmission may or may not occur. Cleri et al (1998a) have studied the problem of fracture when a pre-existing crack lies in the plane of a GB both from the point of view of atomistic simulation and by using a continuum model based on the Rice analysis discussed above. In this paper, we pursue a slightly different problem, and consider the case when a crack, propagating through a perfect crystal, impinges on a GB in its path. We present two different GBs that demonstrate two very different deformation mechanisms, to be described later. The results show the importance of GBs as dislocation sources, and reveal the mechanisms of GB migration due to the stresses ahead of the crack tip. At the same time, the calculations reveal limitations in these sorts of analyses which must be overcome. Namely, the results highlight the need for fully $3 \mathrm{D}$ calculations, as well as the need to understand the dynamics of GB migration and dislocation motion.

This work represents a more detailed, complete analysis of an earlier preliminary set of results dealing with the same set problems (Miller et al 1998). After a brief review of the QC method in section 2 (for a full description, see Shenoy et al (1998)), we present our study of fracture at the atomic scale. The problems investigated in this paper will be divided into the following two sections.

Semi-infinite cracks in single crystals. In this section, our aim will be to use the QC method to compute the critical stress intensity factor to failure for two cracks oriented along different crystal axes. One of these cracks is observed to fail in a brittle manner, by cleavage of atomic planes, while the other is observed to emit a dislocation from the 
crack tip. We compute critical stress intensity values by applying boundary conditions to our model according to the linear elastic solution for the singular portion of the crack-tip fields, and then check that result using a numerical reckoning of the $J$-integral around the crack tip. The resulting critical stress intensity factors are compared with the predictions of two continuum models, and the need for corrections to the continuum models is discussed. Finally, in an effort to shed light on the reasons for the discrepancy between the continuum and atomistic results, we quantify the extent of the nonlinear region around the crack tip. This is achieved both by examining the atomic-level stresses near the crack tip, as well as by comparing the strain fields to those predicted by linear elasticity. We find the nonlinear region to be small (of the order of only a few lattice spacings), but important in light of the error in the continuum approaches. It is also demonstrated that the nonlinear region cannot be well described by a single plane of nonlinearity, which is an assumption often made in continuum models of crack-tip processes.

Interactions between cracks and grain boundaries. Here, we present two simulations of cracks impinging on GBs. In the first case, a $\Sigma=21$ (421) boundary is shown to migrate towards the approaching crack, in addition to emitting dislocations into the bulk crystal regions. The motion of the emitted dislocations is discussed in terms of the elastic interactions between the crack and the dislocations. The motion of the GB can be understood in terms of a continuum model of the configurational force on the GB. We will compute this force and show that its variation along the interface is consistent with the observed GB motion. Finally, we will show that the mechanism of GB motion can be described by the collective motion of the individual dislocations which make up the GB structure. In the second example, a $\Sigma=5(\overline{1} 20)$ boundary proves to be sessile and unable to emit dislocations, and instead the bicrystal fails by intergranular fracture when the crack meets the GB.

\section{Methodology}

In earlier work (Tadmor et al 1996), the QC method was shown to be a viable candidate as an alternative to lattice statics for the treatment of the structure and energetics of defects such as dislocations. The basic idea of this initial treatment was the notion that one can think of an inhomogeneously deformed (and possibly defected) body as a continuum for which the kinematics can be described entirely in terms of displacement fields. Then, rather than supplementing this viewpoint with traditional continuum constitutive models, atomistic analysis is exploited as the basis for determining the total energy of the body. One of the key advantages that emerges from adopting this scheme is the existence of a multiple-well structure to the total energy surface which allows for the stable presence of dislocations.

In a more recent paper, Shenoy et al (1998) presented an amended formulation of the QC method which allowed for the treatment of multiple grains and the correct description of GBs, while remaining essentially equivalent to the original model. Here, we present an outline of this amended logic, while the details of the model can be found in the references mentioned above.

Rather than commencing with a traditional continuum outlook, we adopt the view that a body is composed of some huge number of atoms $N$, and hence that we must at the outset manage $3 N$ degrees of freedom. The goal of the model will be a systematic reduction in these degrees of freedom to reduce the computational demand of the calculations, while retaining the important features of the deformation which arise due to the discrete atomic 
lattice. From the atomistic perspective the total energy can be written as

$$
E_{\text {tot }}=E_{\text {exact }}\left(\boldsymbol{x}_{1}, \boldsymbol{x}_{2}, \ldots, \boldsymbol{x}_{N}\right)=E_{\text {exact }}\left(\left\{\boldsymbol{x}_{i}\right\}\right)
$$

where $\boldsymbol{x}_{i}$ are the atomic coordinates. We see that the total energy depends explicitly on the entirety of the atomistic degrees of freedom that are present.

In order to facilitate a reduction in the number of degrees of freedom, we adopt, as a reference configuration, a purely crystallographic description of the body. The body is assumed to be made up of any number of grains which are defined as non-overlapping polygons. The reference position of each atom within grain number $\mu$ is defined by the triplet of integers $\boldsymbol{l}=\left(l_{1}, l_{2}, l_{3}\right)$ and the expression

$$
\boldsymbol{X}(\boldsymbol{l})=\sum_{a=1}^{3} l_{a} \boldsymbol{B}_{a}^{\mu}+\boldsymbol{R}^{\mu}
$$

where $\boldsymbol{B}_{a}^{\mu}$ is the $a$ th Bravais lattice vector of the $\mu$ th grain and $\boldsymbol{R}^{\mu}$ is the position of one of the atoms in the grain, taken to be its origin atom. The reference and current configurations are related through a set of displacements defined for the entire body as $\boldsymbol{x}=\boldsymbol{X}+\boldsymbol{\psi}(\boldsymbol{X})$. Here capital letters are used to denote measures associated with the undeformed reference configuration and small letters refer to the current configuration. The degrees of freedom are reduced by choosing a set of $R$ representative atoms from the set of $N$ atoms in the body, with $R \ll N$. The positions of these representative atoms remain as the only degrees of freedom in the model, with the positions of the remaining atoms in the body obtained by interpolation. With this in mind, we also define the representative atoms to coincide with the nodes of a finite-element mesh, and thus the interpolated position of any atom is obtained from

$$
\boldsymbol{x}_{i}^{\mathrm{int}}=\sum_{\alpha=1}^{R} N_{\alpha}\left(\boldsymbol{X}_{i}\right) \boldsymbol{x}_{\alpha}
$$

where $N_{\alpha}\left(\boldsymbol{X}_{i}\right)$ is the finite-element shape function centred on the representative atom $\alpha$ and evaluated at the position of atom $i$. This allows us to write the total energy of the body in terms of the interpolated atomic sites as

$$
E_{\text {tot }}=E_{\text {exact }}\left(\boldsymbol{x}_{1}^{\mathrm{int}}, \boldsymbol{x}_{2}^{\mathrm{int}}, \ldots, \boldsymbol{x}_{N}^{\mathrm{int}}\right)=E_{\text {exact }}\left(\left\{\boldsymbol{x}_{i}^{\mathrm{int}}\right\}\right)
$$

where the superscript int refers to the positions of any atom which is not explicitly a node in the mesh, obtained via interpolation. It is worth noting at this point that while the formulation presented in this section is general enough to treat a 3D problem, the implementation used is not fully 3D. The finite-element mesh is $2 \mathrm{D}$, lying in a single crystallographic plane (the $x-y$ plane). Atomic displacements are allowed in the $x, y$, and $z$ directions, but variations in these quantities are only permitted in $x$ and $y$. With reference to traditional lattice statics calculations, this is exactly equivalent to periodic boundary conditions in the $z$-direction, with the minimum periodic length necessary to describe the crystal structure of interest. Fully 3D calculations within the QC framework have been developed elsewhere (Rodney 1998).

The constraint that many of the atoms in the body must obey the interpolated displacement fields succeeds in reducing the degrees of freedom of the problem, but it does nothing to simplify the calculation of the total energy in the body. In order to facilitate the total energy calculation, the additional assumption is made that the total energy of the 
collection of atoms can be decomposed into a sum of well defined site energies associated with each atom. In other words, we assume that

$$
E_{\text {exact }}=\sum_{i=1}^{N} E_{i}
$$

where $E_{i}$ is the site energy of atom $i$. Most atomistic models such as the embedded atom method (EAM) of Foiles et al (1986), and more sophisticated many-body potentials admit of such a decomposition. Note that this assumption is not valid for density functional theory. Because we are modelling crystals of pure nickel, we make use of the EAM site energy for a homonuclear material, which can be written as

$$
E_{i}=\frac{1}{2} \sum_{j(\neq i)} V\left(r_{i j}\right)+F(\bar{\rho})
$$

where $V$ is a pair potential, $r_{i j}$ is the distance between atoms $i$ and $j, F$ is the embedding energy, and $\bar{\rho}$ is the electron density at the site of atom $i$ given by

$$
\bar{\rho}=\sum_{j(\neq i)} \rho\left(r_{i j}\right)
$$

where $\rho\left(r_{i j}\right)$ is the spherically symmetric electron density around an isolated nucleus. The sums in these equations run over all atoms which neighbour atom $i$, to within some cut-off radius $r_{\text {cut }}$.

The calculation of the sum in (5) is analogous to an integral over the entire body in standard finite-element formulations. In order to compute this sum exactly, we are still required to visit each atom $i=1, N$ in the body. The reduction of this sum to an approximate sum over the reduced set of representative atoms is achieved through numerical quadrature, according to which we write

$$
E_{\text {exact }} \approx \sum_{\alpha=1}^{R} n_{\alpha} E_{\alpha}
$$

where the $n_{\alpha}$ are appropriate weights for each representative atom. Physically, these weights can be thought of as the number of atoms in some spatial region around the representative atom which are all assumed to have approximately the same energy. The rules for selecting the $n_{\alpha}$ are such that in the limit for which every atom in a spatial region is chosen as a representative atom (a limit which we refer to as the 'fully refined' limit), then all of these atoms represent only themselves and $n_{\alpha}$ will be unity. On the other hand, in regions where the deformation gradients are slowly varying, the density of representative atoms required to accurately interpolate the displacement fields will be significantly smaller, and consequently the weights for these atoms will be proportionally larger.

At this stage, the calculation of the energy and equilibrium structure of a body composed of $N$ atoms has been reduced to the explicit calculation of the energy of the subset of $R$ representative atoms, with the only unconstrained degrees of freedom in the problem being the positions of these representative atoms. In addition, a final approximation is made in order to speed up the energy calculation of some of the representative atoms. In regions where the deformation gradient is slowly varying, energy calculations can be made more efficiently by assuming that the deformation gradient $\boldsymbol{F}$ is approximately constant. Thus, in these regions a representative atom's site energy is computed by deforming the atomic environment according to the local deformation gradient at the representative atom's site. The Bravais lattice vectors of the deformed configuration, $\boldsymbol{b}_{a}$ are obtained from the reference Bravais lattice via $\boldsymbol{b}_{a}=\boldsymbol{F} \boldsymbol{B}_{a}$, and the uniformly deformed environment of the representative 
atom is uniquely determined. We refer to such atoms, for which the energy is computed from the local deformation gradient, as local representative atoms. On the other hand, in regions where the deformation gradient is rapidly varying on the atomic scale, an atom's site energy must be computed from the fully non-local deformation environment. Non-local representative atoms, then, are atoms for which the local approximation cannot be made. The determination of local or non-local status is made automatically, based on a criterion that examines the variation of the deformation gradient in the vicinity of each representative atom.

The introduction of 'local' atoms into the formulation leads to small spurious forces in the regions where there is a local to non-local transition. These forces, which we refer to as ghost forces, can be corrected in an approximate way, and their effect on final solutions can be made acceptably small. Details about these ghost forces can be found in Shenoy et al (1998).

Once the approximate expression for the total energy is determined through the procedure just outlined, the problem remains only to find minimum energy configurations of the atoms. The procedure used in this paper to minimize the energy is the standard method of conjugate gradients (Press et al 1992), for which we require expressions for the derivatives of the total energy. These details are also explained in Shenoy et al (1998).

Finally, an important aspect of the model is the existence of an adaptive capability by which certain regions of a model are targeted for refinement as deformation fields change in response to changes in loading. In the context of fracture calculations like the ones presented here, this adaption may be triggered due to dislocation activity in the vicinity of a crack tip, or crack propagation due to brittle fracture. The model will allow us to fully refine the region near a crack tip, thus introducing all the atomistic nonlinearity and non-locality necessary in this critical region. Far away from the crack tip, a coarse mesh and local representative atoms will allow for a significant degree of freedom reduction as compared to a fully atomistic model. This will ensure that the crack tip is properly embedded in a bulk elastic crystal whose constitutive behaviour is dictated by the same atomistic model as is used in the crack-tip region. The coarse mesh and local assumption will allow us to model systems which are 'large' on the atomic scale, and at the same time will permit us to employ realistic remote boundary conditions. Furthermore, right at the crack tip, the fully atomistic formulation in this region will permit dislocation activity or crystal cleavage as is dictated by the underlying atomic interactions.

\section{Semi-infinite cracks in single crystals}

In this section, we study the mechanisms of fracture in single crystal, fcc nickel. The vehicle of study is a semi-infinite crack loaded in mode I, which we embed in two different crystal orientations to observe both cleavage and crack-tip dislocation emission. It should be recognized that fcc nickel is not experimentally observed to fracture via cleavage. The fact that we observe this behaviour in our simulations may be attributed to the EAM potentials used to describe this material. For the purposes of our discussion, this material can simply be thought of as a generic fcc metal, for which brittle fracture can be observed at low temperatures.

The crack modelled here is semi-infinite in the sense that the dimension of crack length does not enter into the analysis, but the numerical simulation is of course effected using a finite-sized model. The boundary conditions on this model, as will be described below, are such that the entire mesh can be thought of as only the crack-tip region, embedded in an infinite continuum. 
The goal of these simulations is twofold. First, it is of interest to observe the atomic scale deformation mechanisms which occur at the crack tip when the crack is loaded in mode I. Second, it is possible to use the method to compute critical quantities associated with the deformation processes, and compare them to the predictions of analytic models. We will compute the critical stress intensity factor for either fracture or dislocation emission to occur. This will allow for comparison with models such as Rice's Peierls framework for dislocation emission (Rice 1992), the Griffith energy release rate concept, and the Barenblatt crack, in order to assess the accuracy of these continuum based models.

In addition, we will compute the atomic-level stresses near the crack tip just prior to the critical event with the aim of using these stresses to determine criteria for either cleavage or dislocation emission. We make use of the commonly known virial definition of these stresses (see, for example, Egami and Srolovitz (1982) or Vitek and Egami (1987)), which for the EAM description of a material can be shown to be

$$
\sigma_{i}^{I J}=\frac{1}{\Omega_{i}} \sum_{j \neq i}\left[V^{\prime}\left(r_{i j}\right)+F^{\prime}\left(\bar{\rho}_{i}\right) \rho^{\prime}\left(r_{i j}\right)+F^{\prime}\left(\bar{\rho}_{j}\right) \rho^{\prime}\left(r_{i j}\right)\right] \frac{r_{i j}^{I} r_{i j}^{J}}{r_{i j}}
$$

where the superscripts $I$ and $J$ refer to Cartesian components, $\sigma_{i}^{I J}$ refers to the stress tensor at atom $i, r_{i j}^{I}$ is the $I$ th component of the relative position vector between atoms $i$ and $j$, and $r_{i j}$ is its magnitude. $\Omega_{i}$ is the atomic volume at site $i$, and the remaining quantities are as previously defined for EAM (a prime denotes differentiation). This definition of the stresses will only agree with the continuum definition in the case of uniform strains in a bulk crystal, and it has been recognized (Cheung and Yip 1991) that the virial definition can be significantly different from the continuum stress near free surfaces. In light of this fact, we must exercise caution in making any direct comparisons between the continuum and atomic-level definitions of stress. We will examine the agreement between the atomic-level stresses near the crack tip and the crack-tip fields of linear elasticity. Later, we will compare the critical atomic-level stresses to simple estimates of the theoretical strength of crystals.

\subsection{Cracks in single crystals: geometry and loading}

A schematic of the geometry used in these simulations is shown in figure 1. The two crystallographic orientations and accompanying mesh dimensions are presented in table 1, as defined by the labels in the figure. For reasons that will become apparent later, we will refer to these as the brittle and ductile crystallographic orientations. Note that the cracks we model are not ideally sharp, but have a finite opening corresponding to the removal of one plane of atoms prior to loading. All cracks will be remotely loaded in mode I. In this section, we are interested only in studying a single critical event at a crack tip-either the emission of a dislocation or the propagation of a crack. Hence, the simulations in this section do not make use of mesh adaption. The initial mesh is chosen to be fully refined near the crack tip, and gradually becomes more coarse away from the fracture process zone.

Table 1. Details of single crystal crack models. All dimensions are in angstroms and refer to figure 1.

\begin{tabular}{lllllll}
\hline Crack & $x$-axis & $y$-axis & $a$ & $h$ & $w$ & $d$ \\
\hline Brittle & $(110)$ & $(001)$ & 1912 & 3604 & 3823 & 3.52 \\
Ductile & $(111)$ & $(1 \overline{1} 0)$ & 2601 & 5735 & 5203 & 2.489 \\
\hline
\end{tabular}




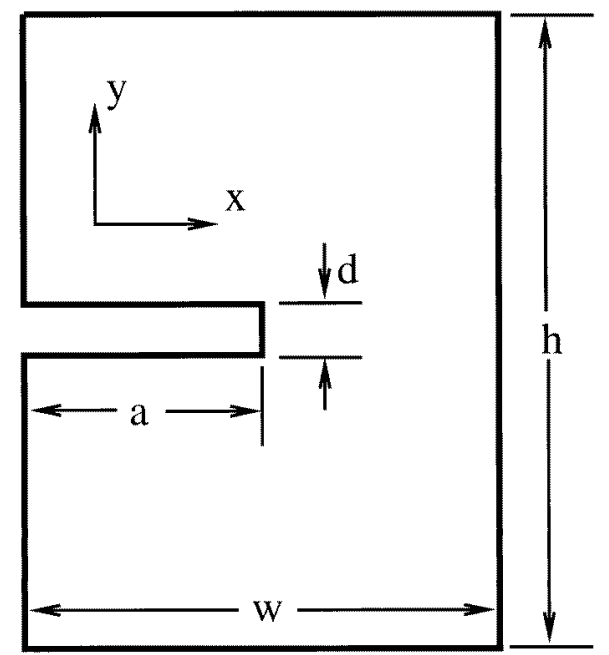

Figure 1. A schematic diagram of the crack geometry considered, indicating the important dimensions.

The most natural way to quantify the critical load for brittle or ductile response is by making use of the fracture toughness concept of linear fracture mechanics. As we shall explain later, our loading conditions are such that the boundaries are held fixed to displacements corresponding to the anisotropic, linear elastic solution for a sharp crack. Thus, for each load, the displacements at the boundaries correspond to a certain value of $K_{I}$. On the basis of these remotely applied displacement fields, we can compute the critical value of $K_{I}$ at which either dislocation emission $\left(K_{I C}^{\mathrm{d}}\right)$ or crack advance $\left(K_{I C}^{\mathrm{c}}\right)$ occurs. The former can be compared to the continuum-based model of dislocation emission put forth by Rice (1992), while the latter corresponds to the Griffith fracture toughness of the material for the crystal orientation being considered.

In order to accurately compute $K_{I C}^{\mathrm{d}}$ or $K_{I C}^{\mathrm{c}}$ for a certain geometry, the following strategy is devised. Based on the large model size (see table 1), it is assumed that the fields at the boundaries can be well approximated by those from linear elasticity, and for each load step the boundary node displacements are fixed to these fields. Even for a crack of small but finite bluntness (of the order of a few angstroms), we will assume that the crack is ideally sharp as far as the model boundaries are concerned. Note also that while the dimension $a$ has been included in table 1, it enters the analysis only insofar as we have assumed that this distance is far enough from the crack tip for the edges of the model to obey linear elasticity exactly. All of the boundaries except the crack faces themselves will be held fixed to the crack-tip displacement fields of linear elasticity, and thus it is as though our mesh were embedded in an infinite body containing a semi-infinite crack.

The critical value of $K_{I C}$ at which inelastic deformation (i.e. either crack advance or dislocation activity) occurs can be computed by gradually increasing the displacements at the boundaries according to the linear elastic fields, and allowing the internal nodes of the model to relax at each load step. Problems arise, however, due to the non-convex nature of the potential energy surface that dictates the crystal behaviour. Taking load steps which are too large, or relaxing from an initial configuration that is very far removed from the minimum energy configuration can lead to different solutions. This can be avoided by quasistatic loading, i.e. by taking small load steps. However, this is still not practical since 
the number of steps required and the resulting computational time quickly become large.

We have devised the following method for obtaining accurate minimum energy configurations within realistic computational time. We define $\boldsymbol{u}\left(\boldsymbol{X}, K_{I}\right)$ to be the displacement fields which correspond to a sharp crack in an anisotropic linear elastic material loaded in mode I (see, for example, Sih and Liebowitz (1968)). The fields $\boldsymbol{u}\left(\boldsymbol{X}, K_{I}\right)$ are functions of the position $\boldsymbol{X}$ and the strength of the field, given by the stress intensity factor, $K_{I}$. We can then use this field for the initial condition of our simulation. We expect that far from the crack tip, this will be the minimum energy solution, while nodes close to the crack tip will want to rearrange as atomic effects become more important. The initial displacements of the mesh for the first load step, denoted by $\boldsymbol{\psi}_{0}^{\text {init }}\left(\boldsymbol{X}, K_{I, 0}\right)$, are given by

$$
\boldsymbol{\psi}_{0}^{\text {init }}\left(\boldsymbol{X}, K_{I, 0}\right)=\boldsymbol{u}\left(\boldsymbol{X}, K_{I, 0}\right)
$$

where $\boldsymbol{X}$ is any point in the model and $K_{I, 0}$ is some initial stress intensity factor, chosen to be small enough to ensure that even those atoms close to the crack tip will have positions that are similar to those dictated by the elastic solution. We then freeze the boundary nodes at these displacements, and allow the internal nodes to relax via a conjugate gradient minimization of the total potential energy. The resulting displacement field $\psi_{0}\left(X, K_{I, 0}\right)$ is the minimum energy configuration for the current boundary loading. For subsequent load steps, rather than start from the linear elastic field each time, we instead add the additional linear elastic displacements that correspond to increasing the stress intensity factor by an amount $\Delta K_{I}$. By the linearity of the elastic fields, the additional displacement is just $\boldsymbol{u}\left(\boldsymbol{X}, \Delta K_{I}\right)$, and hence the initial conditions for each subsequent load step is given by

$$
\boldsymbol{\psi}_{n}^{\text {init }}\left(\boldsymbol{X}, K_{I, n}\right)=\boldsymbol{\psi}_{n-1}\left(\boldsymbol{X}, K_{I, n-1}\right)+\boldsymbol{u}\left(\boldsymbol{X}, \Delta K_{I}\right)
$$

where $K_{I, n}=K_{I, 0}+n \Delta K_{I}$. We then allow this field to relax to the minimum energy displacement field, $\boldsymbol{\psi}_{n}\left(\boldsymbol{X}, K_{I, n}\right)$. This method allows us to take larger steps than could be taken by simply displacing the boundaries nodes, but provides a substantially better initial guess than linear elasticity near the crack tip. Our solution procedure amounts to the assumption that if the increment in the stress intensity factor between two load steps is small, then the change in the nonlinear part of the displacement field will also be small, and the initial guess will therefore be a good approximation to the final solution. Note that because the boundary nodes are always held fixed during relaxation, their displacements will correspond to the $K_{I}$ fields exactly, and thus the model will behave as though it were embedded in a larger continuous body subjected to remote loading. In addition, since a given boundary displacement corresponds to a known value of $K_{I}$, it can be used to determine the critical stress intensity factor.

The algorithm just described would still require a large number of load steps in order to obtain $K_{I C}$ to any reasonable accuracy. We further improve our solution algorithm by taking advantage of the unstable nature of crack-tip deformation processes and vary the size of our load steps, $\Delta K_{I}$. During the minimization process for a given load step, we periodically monitor the deformation within each element in the fully refined region near the crack tip. These elements have three atoms as their nodes, any two of which define an atomic plane with the third residing on a neighbouring plane. By comparing the reference and current configurations of these atoms, we can compute a local value of the slip and opening which has taken place between these two atomic planes. For example, the fully refined region near a crack tip is shown in figure 2 in both the reference and deformed configurations. By comparing the two configurations for the highlighted element, we can estimate the slip between the (111) planes in the [110] direction. By our knowledge of the expected slip and decohesion processes in fcc crystals, we can identify critical values for 
slip and opening which signify that a dislocation has been emitted or that the crack has advanced (for example, we know the length of the Shockley partial Burgers vector to be $b=1.437 \AA$ in fcc nickel). Thus, if we find that slip or opening has occurred, we know that the current value of $K_{I}$ is above the critical value. The current load step is then aborted, the deformation field of the last successful $K_{I}$ value is restored, and a smaller load step is taken. By taking large load steps until the critical load is approached, and then by taking smaller and smaller load steps until the current load is within some tolerance of the critical load, the critical value $K_{I C}$ can be found quickly and accurately.

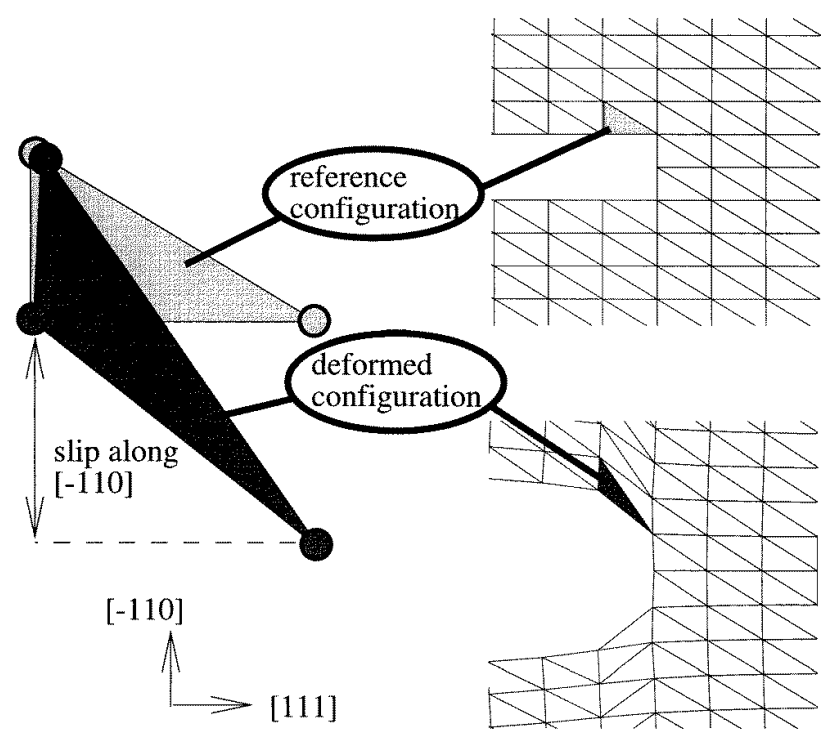

Figure 2. Computing elemental values of crystalline slip. By comparing the reference and deformed configuration in the fully refined region near a crack tip, the local value of slip along the $[\overline{1} 10]$ direction between the (111) planes is computed.

As pointed out by Gumbsch (1995), the anisotropic solution used to determine the boundary condition in this simulation is only exact for the brittle crystal orientation. In the ductile orientation, the solution is only approximate because the $x-y$ plane of the model is not a plane of elastic symmetry. In an effort to quantify the level of this error, and check the self-consistency of our approach, we will take advantage of the finite-element underpinnings of the model to numerically evaluate the path independent $J$-integral (Rice 1968) associated with each of the crack tips in these models. The $J$-integral yields the energy release rate, $G$, associated with a given crack geometry. We will make this calculation using the area integral method of DeLorenzi (1982), the implementation of which is discussed by Shih and Moran (1987).

\subsection{Cracks in single crystals: failure mechanisms and comparison to theoretical models}

Figure 3 shows the failure mechanisms for the two orientations. Contours in the figure are of elemental slip, as described in figure 2. For each element, the three slip planes defined by each pair of nodes are considered, and the maximum of the slip on these planes is plotted. The results in figure 3 show the crack tip for a load just below the critical load, as well as for a load just above the critical value. In the brittle orientation, we see the crack fails by 


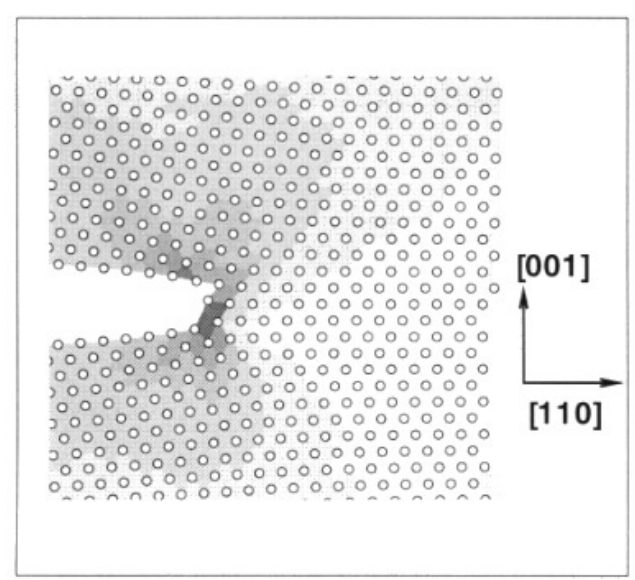

(a)

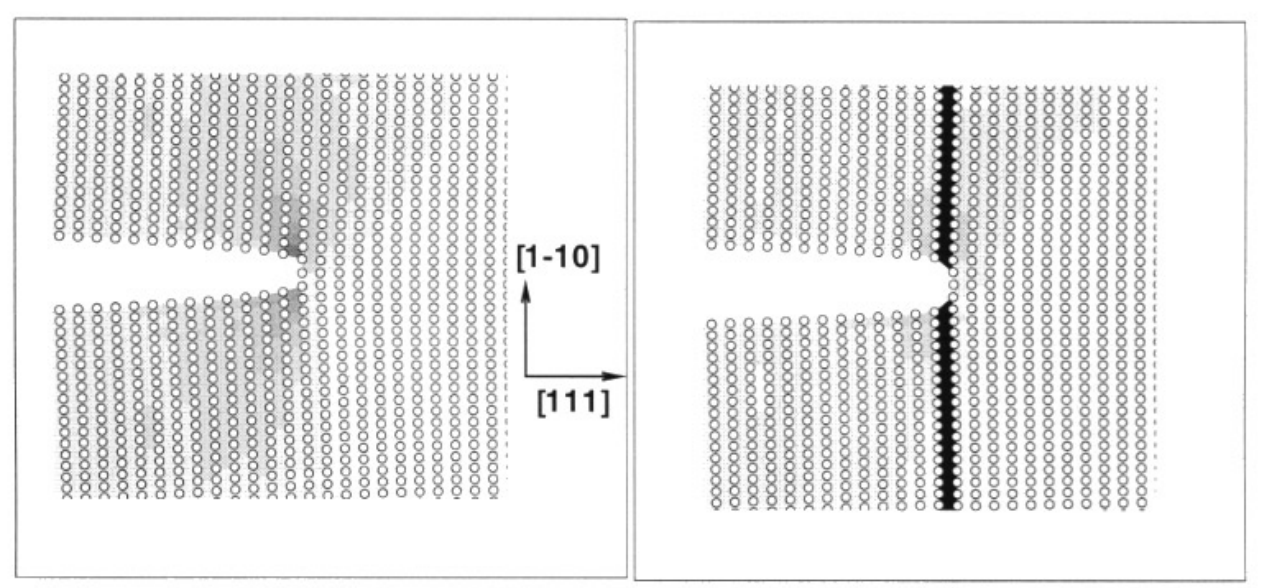

(c)

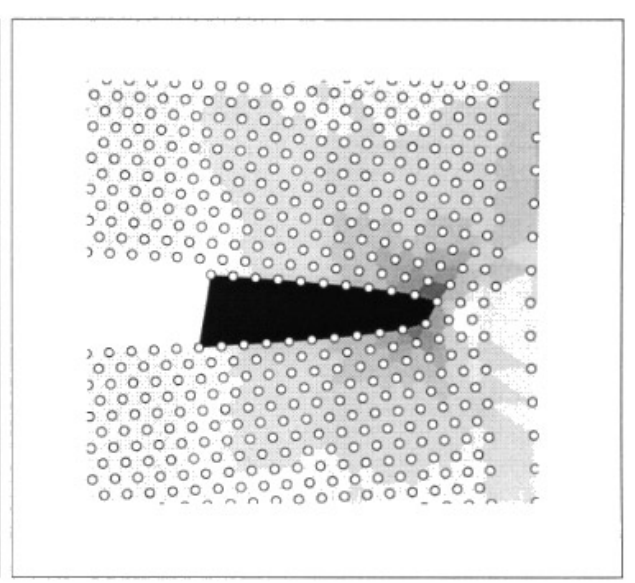

(b)

Figure 3. Two different failure mechanisms in nickel. (a) is the brittle crack orientation, just before cleavage occurs, while (b) is the next load step. (c) and (d) show the ductile orientation, before and after the emission of two Shockley partials. Contours between atoms are of elemental slip, as described in the text, and are shown to draw attention to the slip traces in (d).

advancing into the crystal. On the other hand, the ductile orientation fails when the crack tip is blunted by the emission of two Shockley partial dislocations normal to the crack plane.

Using the procedure outlined in the previous section, the $K_{I C}$ values for the two semiinfinite cracks were computed. The results are summarized in table 2 , where they have also been converted to the equivalent values of the energy release rate $G$ (see Sih and Liebowitz 1968), which are compared to the numerically computed $J$-integrals around the crack tips.

In the following discussion of the results in table 2, we will focus on the values of $G$ and $J$, keeping in mind that $K_{I C}$ and $G$ are obtained from the analytic solution corresponding to the model boundary displacements, and are essentially two representations of the same quantity. The agreement between $G$ and $J$ for the brittle simulation where the elastic solution is rigorously correct is about $6 \%$. This lack of exact agreement between the analytic and numerical values is typical of numerical computations of the $J$-integral in 
Table 2. Results of single crystal semi-infinite crack models. Units of $K_{I C}$ are $\mathrm{MPa} \mathrm{m}^{1 / 2}, G$ and $J$ are in units of $\mathrm{J} \mathrm{m}^{-2}$. The values of $K_{I C}$ and $G$ are obtained from the analytic solution in accordance with the prescribed boundary displacements, while $J$ is computed numerically from the relaxed solutions.

\begin{tabular}{lll}
\hline Orientation & $K_{I C} \Longleftrightarrow G$ & $J$ \\
\hline Brittle & $0.4898 \Longleftrightarrow 3.337$ & 3.536 \\
Ductile & $0.3954 \Longleftrightarrow 2.326$ & 2.592 \\
\hline
\end{tabular}

which the mesh is not optimally designed for this purpose (Shih and Moran 1987). Because the calculation of $G$ is essentially exact in the brittle orientation, the $6 \%$ error between $G$ and $J$ may be thought of as providing error bars on the $J$-integral calculation. Perhaps it is more important to simply note that despite the approximations, the discrepancies between the two calculations are only a few per cent-which is reasonable for the qualitative discussion which we will present later. For the remainder of this section, we will take $G$ as the best estimate of the energy release rate experienced by the crack tips.

The calculation of atomic-level stresses according to equation (9) was performed for each crack just prior to the critical event, and is plotted in figure 4. Figure 4(a) is taken from the brittle orientation, and shows $\sigma^{y y}$, along the $x$-direction directly ahead of the crack tip. Figure 4(b) is for the ductile orientation, and shows the resolved shear stress $\tau$ tending to form a Shockley partial dislocation, along the positive $y$-direction starting from the crack tip. For comparison, the elastic $1 / \sqrt{r}$ curve is also presented in each figure. This elastic curve is simply a best fit to the actual stresses beyond the first three lattice spacings from the crack tip. The same curve can be generated from the actual applied $K_{I}$ field, but the atomically blunt crack makes the location of the origin ambiguous. Note that away from the crack tip, the $1 / \sqrt{r}$ form is closely matched, but both plots deviate significantly in the region immediately around the crack tip where nonlinear effects become important. The fact that the shear stress in figure 4(b) shows a more marked deviation from the elastic curve than the tensile stress of figure 4(a) is due to the existence of an incipient slip distribution along the line for which the stress is being plotted. The maximum value of the tensile stress at the tip in figure 4(a) corresponds to the critical stress required to break the first atomic bond at this point, and thus initiate the propagation of the crack. This maximum value is approximately $\sigma_{\mathrm{c}}^{y y}=32.0 \mathrm{GPa}$. The maximum shear stress in figure 4(b), right at the crack tip, is approximately $\tau_{\mathrm{c}}=5.6 \mathrm{GPa}$. For comparison, we note that $C_{11}=233.0 \mathrm{GPa}$ for this material.

These results make possible comparisons to analytic models that seek to predict the same critical values. Here, we examine two such approaches. The first is the model proposed by Rice (1992), in which brittle or ductile behaviour is viewed as a competition between Griffith fracture and dislocation emission at the crack tip. For Griffith fracture, the critical quantity is an energy release rate $G_{\mathrm{c}}$ equal to twice the surface energy associated with the newly created surfaces. For the emission of a dislocation, on the other hand, the critical quantity is the unstable stacking fault energy, which is the maximum of the interfacial energy when two halves of a crystal are rigidly displaced with respect to one another along the slip direction. By taking into account the orientation of the slip direction with respect to the crack plane, this emission criterion can also be stated in terms of an energy release rate, $G_{\mathrm{d}}$, and thus the lower of $G_{\mathrm{c}}$ and $G_{\mathrm{d}}$ will determine the predicted behaviour. Second, we will compare the critical values of the atomic-level stress from the simulation with those obtained from the calculation of the ideal strength of a perfect crystal. The ideal shear strength is defined, 

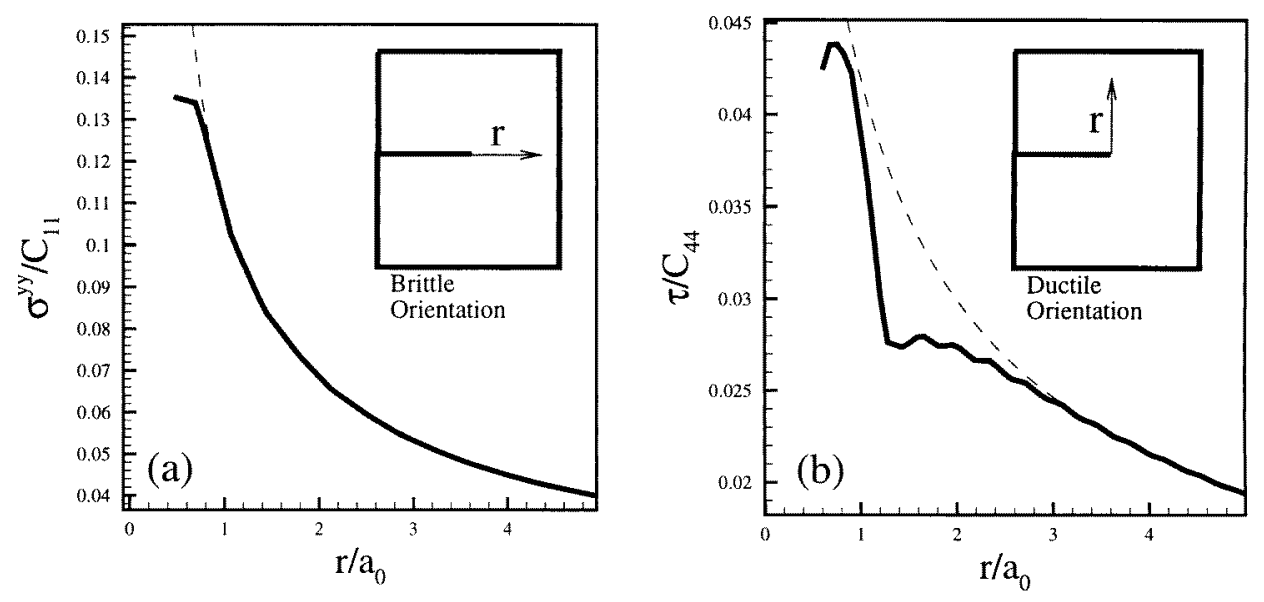

Figure 4. Atomic-level stresses for two different orientations of the semi-infinite crack. (a) shows the stress prior to crack propagation in the brittle orientation, while (b) is prior to the emission of a Shockley partial in the ductile orientation. The full curve is extracted from the simulation, while the dashed curve is the elastically predicted $1 / \sqrt{r}$ curve. Stresses are normalized by the elastic constants $C_{11}=233.0 \mathrm{GPa}$ and $C_{44}=128.0 \mathrm{GPa}$.

for our purposes, as the maximum stress required to rigidly shear two halves of a crystal with respect to one another, while the ideal tensile strength is the maximum stress required to pull those two rigid half crystals apart. Each of these quantities can be obtained from a simple atomistic calculation as the maximum derivatives of the $\gamma$-surface (Vitek 1968) and universal binding energy relation (UBER) (Rose et al 1981) for the crystallographic plane of interest.

In table 3, we compare the theoretical predictions for the material response with the results of our simulations. In the first two columns, the critical values of $G_{\mathrm{c}}$ and $G_{\mathrm{d}}$ for crack advance and dislocation emission from the Rice model are shown. These are compared with column three, where the actual value of $G$ from the simulation is presented. All of the theoretical values in this table were obtained by performing separate atomistic calculations. Where appropriate, relaxations of the atomistic unit cell were permitted perpendicular to the slip plane. A Poisson's ratio of $v=0.33$ was used in the Rice analysis.

Table 3. Comparison between the simulations and the theoretical predications. The values of $G$ are in units of $\mathrm{J} \mathrm{m}^{-2}$. The strength values are in units of GPa, and refer to different stress components, as appropriate to the observed failure mechanism. We present $\sigma^{y y}$ for the brittle orientation and $\tau_{\mathrm{c}}$ for the ductile orientation.

\begin{tabular}{llllll}
\hline Orientation & $G_{\mathrm{c}}$ & $G_{\mathrm{d}}$ & $G$ & $\begin{array}{l}\text { Theoretical } \\
\text { strength }\end{array}$ & $\begin{array}{l}\text { Actual } \\
\text { strength }\end{array}$ \\
\hline Brittle & 3.140 & 2.943 & 3.337 & 27.9 & 32 \\
Ductile & 3.461 & 1.252 & 2.326 & 2.4 & 5.6 \\
\hline
\end{tabular}

Notice from table 3 that the Rice model yields $G_{\mathrm{d}} / G_{\mathrm{c}}<1$ for both orientations, thus predicting that both should exhibit a ductile response. In the ductile orientation, while this prediction is qualitatively correct, the Rice model still underestimates the actual value of $G$ at emission by about $50 \%$, which is consistent with observations made by a number of other 
authors (see, for example, Zhou et al (1993)). In the brittle orientation, the competition between cleavage and dislocation emission is subtle (probably closer than the accuracy of the models themselves). In this case, the Rice model is qualitatively incorrect and predicts dislocation emission. At the same time, however, $G$ and $G_{\mathrm{c}}$ are in very good agreementwith $G$ being about $6 \%$ greater than $G_{\mathrm{c}}$. The fact that $G_{\mathrm{c}}$ slightly underpredicts the energy release rate for brittle fracture is at first surprising, since the energy balance for perfectly brittle fracture should be exact in this case. The error can be explained, however, by the atomic scale bluntness of our crack tip. Recall that the cracks are produced by removing a single atomic layer. The tendency for $G$ to increase with crack-tip bluntness has been observed by Gumbsch (1995), and studied in detail by Schiotz et al (1997). On the other hand, the value of $G_{\mathrm{d}}$ in the brittle orientation is again too low. Indeed, if we use as a rule of thumb the $50 \%$ error observed in the ductile orientation just discussed, we can compute a corrected value for dislocation emission that restores the qualitative agreement with the simulations.

It is worth noting that a number of authors have sought simple corrections to the Rice framework that can improve the accuracy of the $G_{\mathrm{d}}$ prediction. For example, Zhou et al (1994), Thomson and Carlsson (1994) and Juan and Kaxiras (1996) have all proposed ways to include the effect of the atomic scale ledge which forms at the crack tip during blunting. The sign of the corrections they obtain is consistent with our results, in that the ledge effect increases the value of $G_{\mathrm{d}}$. In another example, Gumbsch and Beltz (1995) have examined the same crystallography and material as our brittle orientation, and found the Rice model to correctly predict the brittle response. In this case their model included the so-called shear-tension coupling effect in the dislocation emission process, rather than any correction due to ledge energy. It is not clear, however, that any of these models include all of the relevant effects near the crack tip. Indeed, we will show later that the extent of the nonlinear zone prior to failure is greater than a single atomic plane. This is at odds with the simplifying assumption of most continuum-based models, whereby all nonlinearity is confined to a planar cohesive zone.

In the last two columns of table 3, we compare the maximum value of the appropriate component of the atomic-level stress tensor with the theoretical strength as defined previously. In the brittle orientation, the comparison is between the maximum value of $\sigma^{y y}$ and the theoretical tensile strength. These values compare reasonably well, with an error less than about $15 \%$. For the orientation which leads to dislocation emission, the appropriate comparison is between the critical resolved shear stress tending to produce the first Shockley partial and the theoretical shear strength. In the case, the agreement is considerably worse, with the theoretical shear strength being more than $50 \%$ lower than the observed atomic-level stress. Given that the assumptions used in deriving the theoretical strength values are closely related to those used in determining $G_{\mathrm{d}}$ and $G_{\mathrm{c}}$ in table 3 , it is perhaps not surprising that the case leading to cleavage is in fairly good agreement while in the dislocation emission case the agreement is poor. This result further emphasizes the need for some correction to the treatment of shear deformation and dislocation emission.

We have computed critical values of the energy release rate for brittle fracture and dislocation emission from our atomic-level simulations. Using these values as benchmarks, we have examined the ability of a number of continuum-based analyses to predict these critical values. We have found that these models are unable to accurately predict critical loads to failure. In the next section, we will provide evidence for what may be one of the factors contributing to the error in the continuum framework. An essential feature of the Rice model and its derivatives is the assumption of a cohesive zone. Essentially, the model is linear elastic, but for a single mathematical plane between two layers of atoms that defines 
this zone. The tractability of the model arises from the assumption that all nonlinearity is confined to the cohesive zone, and linear elasticity can be used for the remainder of the body. In the next section, we examine this assumption by computing the extent of the region in which nonlinear effects are substantial. We measure this nonlinearity by comparing the strains near the crack tip to strains predicted from a purely linear model.

\subsection{The degree of nonlinearity near the crack tip}

In order to determine the degree of nonlinearity near the crack tip, we have modelled the semi-infinite cracks with the same mesh of constant strain triangular elements as was used in the QC solution, but now with an anisotropic linear elastic constitutive law. The goal of this calculation is a direct comparison of the strain fields resulting from the two solutions. The use of identical meshes and elements ensures that errors in the solution due to mesh effects are the same for both the QC and linear elasticity result, and therefore will not affect our comparison. The linear elastic solution is obtained using the generalized Hooke's law in order to include the full anisotropy of the fcc nickel, while the moduli used are those obtained from the EAM potentials which underlie the QC model. The finite-element formulation for both models is for finite strain, and thus the linear elasticity model is based on the assumption that the strain energy density can be written as

$$
\mathcal{E}=\frac{1}{2} C_{i j k l} \epsilon_{i j} \epsilon_{k l}
$$

where $C_{i j k l}$ are the moduli and $\epsilon_{i j}$ are the components of the Lagrangian finite strain tensor. In figure 5, we present the difference between the strain fields obtained using QC and those using the linear elasticity model. The comparison is made by computing the fields for the same value of boundary displacements, and allowing all interior nodes to move to their minimum energy configuration. The plots are for boundary loads which correspond to values of $G$ just below the critical value in each orientation. Thus, we are examining the validity of linear elastic analysis just before the onset of inelastic (i.e. fracture or dislocation motion) deformation. Note that these plots are of, for example, $\epsilon_{x x}^{\mathrm{QC}}-\epsilon_{x x}^{\mathrm{LE}}$. This is the amount by which the strain that occurs in the atomistic model differs from the strain of the linear elasticity model, and can be thought of as the strain that is missed by using the linear elastic constitutive model. Because the elements are constant strain triangles, nodal values of strain are computed by averaging over all elements that a node touches. It is these nodal strains that are compared in the figure. Directly on the figure, values shown indicate the location and magnitude of the maximum and minimum strain differences. To put these levels into proper context, we note that the linear elastic solution for the brittle orientation yields the following maximum values of strain near the crack tip: $\left.\epsilon_{x x}^{\mathrm{LE}}\right|_{\max }=0.083,\left.\epsilon_{y y}^{\mathrm{LE}}\right|_{\max }=0.213$, and $\left.\epsilon_{x y}^{\mathrm{LE}}\right|_{\max }=0.063$. Corresponding maxima in the ductile orientation are $\left.\epsilon_{x x}^{\mathrm{LE}}\right|_{\max }=0.071$, $\left.\epsilon_{y y}^{\mathrm{LE}}\right|_{\max }=0.181$, and $\left.\epsilon_{x y}^{\mathrm{LE}}\right|_{\max }=0.097$.

The plots suggest that the linear elastic fields are actually quite representative of the QC result, up to a small region near the crack tip of only about $10 \AA$ in radius. An exception to this is the effect of surface relaxation on the crack faces in the ductile orientation, which can be seen in the plot of $\epsilon_{y y}$. Surface relaxations aside, such good agreement between atomistic and linear elasticity models suggests that the nonlinear zone surrounding an atomic scale crack tip is surprisingly small, which agrees with similar observations made by other authors (Cleri et al 1998b). Nonlinear effects become significant only very close to the tip of the crack. However, it is clear that because the critical event occurs due to the breaking of bonds at the crack tip, an accurate characterization of the nonlinear region is essential. Furthermore, it is evident from the figure that nonlinearity is not confined to a single atomic 


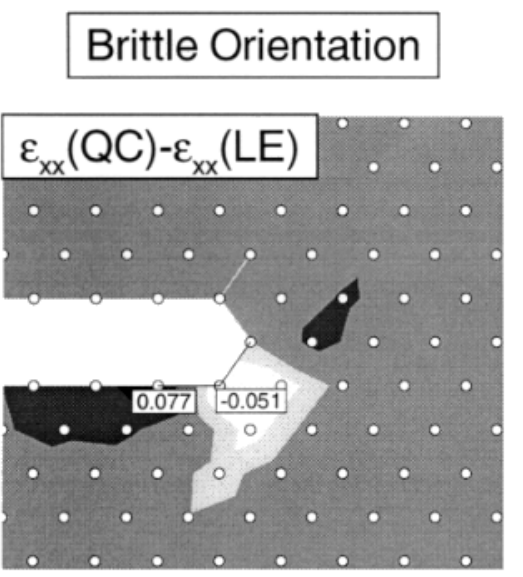

\section{Ductile Orientation}
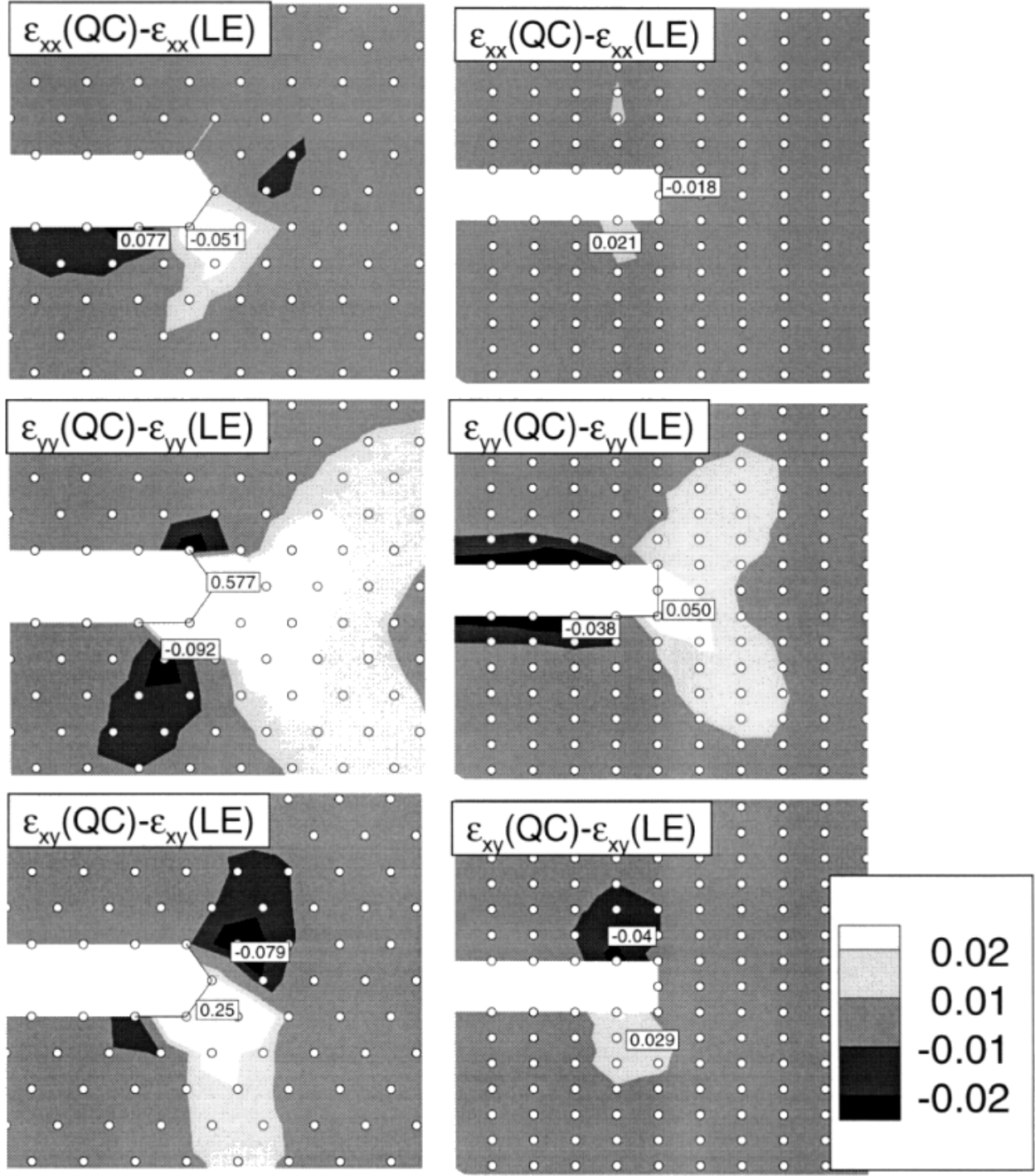

Figure 5. Plots of the difference in strains between the linear elastic and QC models, shown in the reference configurations. The most lightly shaded regions show excess tensile strains greater than $2 \%$, while the darkest regions show excess compressive strains less than $-2 \%$.

plane at the tip of the crack, as postulated in the cohesive zone approach. This observation points to the need for more extensive nonlinear zones in continuum modelling of crack-tip deformation.

Finally, it is worth discussing in somewhat more detail the case of the $\epsilon_{y y}$ strains in the brittle orientation. Here, we note that there is a significant nonlinear region, and the effect of the nonlinearity is to increase the magnitude of $\epsilon_{y y}$ compared to the linear elastic prediction. This observation is consistent with the ideas of Abraham et al (1998), who 
proposed that the uniaxially expanded lattice is a more accurate description of the local atomic environment ahead of the crack then the simple Griffith picture of rigid separation of two atomic planes. The snapshot of the strain field just prior to failure that we present in figure 5 indeed supports this notion.

\section{Interactions between cracks and grain boundaries}

We now turn to the problem of interactions between cracks and grain boundaries. Mechanistic understanding of the role of GBs in fracture of polygranular materials requires a correct atomic-level description of both the advancing crack tip, and the structure of the GB itself. The QC formulation was extended to treat polycrystals by Shenoy et al (1998), and in the same work it was demonstrated that the method predicts GB structures in excellent agreement with fully atomistic models. Thus, in this section, we couple the accurate modelling of GB structure with the ability to model fracture processes demonstrated in the previous section. This is a problem whose size scale is well suited to the QC method, as multiple length scales are clearly at work. These length scales range from those needed to capture the elastic differences between the two grains to the detailed atomistic mechanisms of fracture, dislocation generation, and GB migration.

\subsection{Grain boundary migration due to the approach of a crack}

The first nickel bicrystal we consider contains a $\Sigma=21(421)$ symmetric tilt boundary, which has a [112] tilt axis and a tilt angle of $44.41^{\circ}$. A crack is initiated in the model by removing a single (111) plane from one of the grains. The crystallography of this GB dictates that such a (111) plane is oriented at an angle of $67.8^{\circ}$ to the GB, and we orient the two crystals such that the crack plane lies on the $x$-axis and the GB extends obliquely through the model. The initial mesh is shown in figure 6, with a close-up of the fully refined crack-tip region in the inset. This fully refined region is entirely non-local but for a thin pad of local nodes around its exterior. A load is applied using the method outlined in section 3, except that the isotropic linear elastic crack-tip fields are used. The use of the isotropic solution is necessary because the analytic solution for the crack-tip fields near an oblique plane of moduli discontinuity is not known. Since our model is neither isotropic nor homogeneous, this approach will not provide any quantitative information about stress intensities or energy release rates. The scheme is merely an efficient method of loading the model. Ten load steps were computed, the first of which has zero external load. This first step was necessary to allow the GB to relax to its equilibrium configuration before the approach of the crack.

Adaptive meshing is included in this run in a limited way, due to two complicating factors. First, the existence of a GB in the model means that there will be some point where the full, atomistic detail of the GB structure is truncated. If we do not truncate the GB in this way, it will be necessary to include a fully refined mesh along the entire length of the GB in the model, and our ability to significantly reduce the degrees of freedom of the problem will be reduced. In addition, far from the crack tip it is expected that the GB will behave in an elastic fashion, making this atomic scale information unimportant. Thus, we are left to contend with an abrupt end to the atomic-level resolution of the GB. This point will normally trigger adaption due to the jump in the deformation that arises from the truncation, and hence a wave of adaption will tend to move along the GB. A second complicating factor is that there is the potential for a great deal of dislocation activity in the vicinity of the crack tip. To achieve a true equilibrium, these dislocations will want to run 


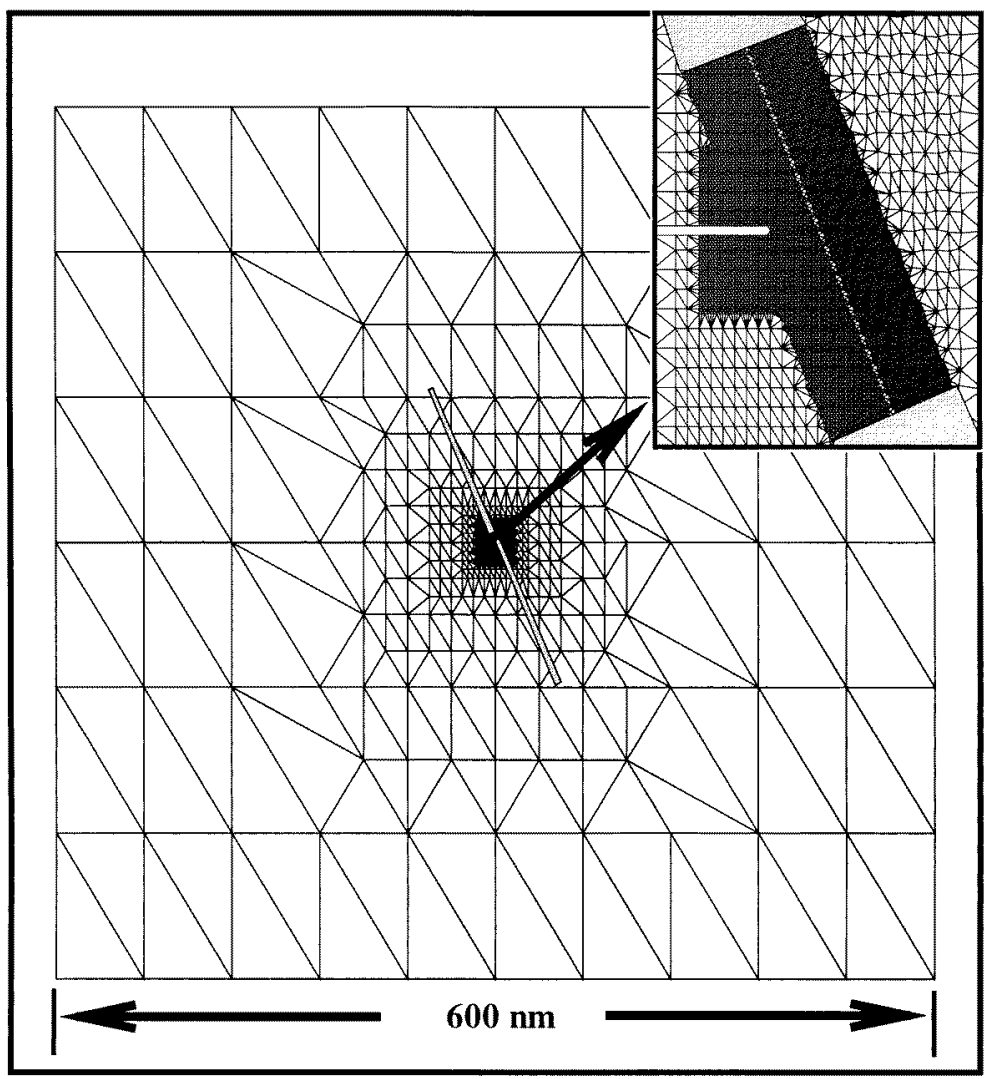

Figure 6. Mesh used in the QC model of the interaction between a crack and a GB. Approximate dimension is shown, and the inset shows a close-up of the fully refined region near the crack tip. The regions in the mesh where adaption is not allowed to take place are shown schematically by the shaded grey boxes.

far from the crack-tip region, thus requiring a very long simulation time and a high level of adaption to follow these advancing dislocations. As we shall see later, the equilibrium distance for these dislocations is of the order of thousands of angstroms. The first of these problems is avoided by defining regions where adaption is not allowed to take place-in this case these regions are two narrow bands extended along the GB beyond the fully refined region, as shown schematically in figure 6 . The second problem is dealt with by adapting only three times for each load step, re-establishing equilibrium between each adaption. The assumption here is that this will allow dislocations to travel far enough that their influence on further deformation near the GB is negligible.

Figure 7 focuses on the atoms in the fully refined region for various load steps (with some atoms in the surrounding coarsened region also visible). The loading is quasistatic, and the time parameter in each frame is merely used to indicate the relative magnitude of the loads, as each load step corresponds to the same increment in boundary displacements. Contours between the atoms are contours of elemental slip in the plane of the figure, as described previously, and help us to trace the paths of dislocations as they move through the crystal. Figure 7(a), at $t=1$, shows the initial positions of the crack and GB. At this point, the GB has rearranged to its minimum energy configuration, while the bulk crystals 


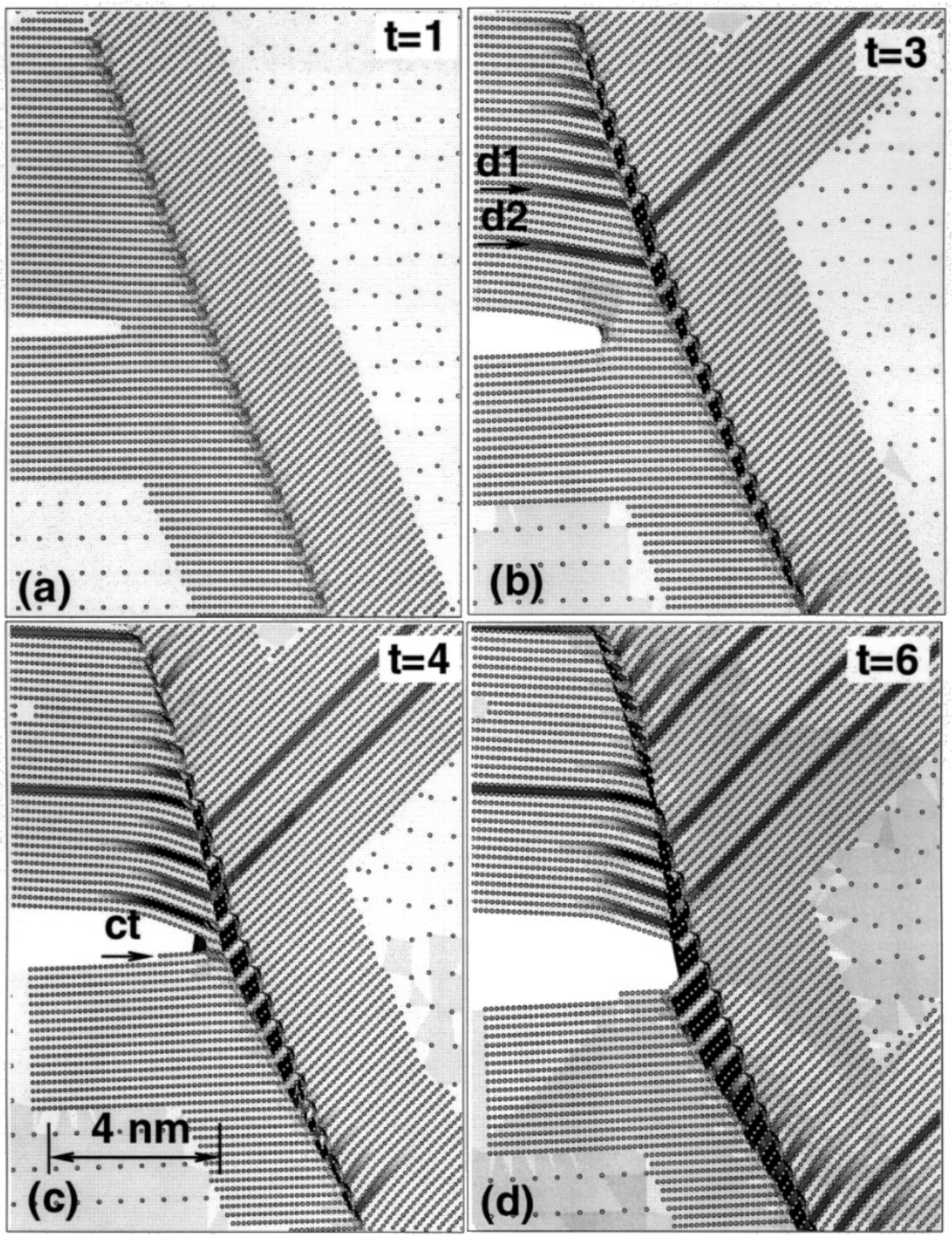

Figure 7. Simulation of a crack running up to a $\Sigma=21(421) \mathrm{GB}$. The width of view in each frame is approximately $100 \AA$. See the text for details.

are still only subject to small strains.

Figure 7(b) depicts the solution after the third time increment, where we see that a number of dislocations have been emitted from the GB above the plane of the crack. The slip traces of these dislocations appear as dark bands between atomic planes. The 
dislocation line lies out of the plane of the figure, while the core of the dislocation is the region where each slip trace dies out. Two prominent dislocations are labeled $\mathrm{d} 1 \mathrm{and} \mathrm{d} 2$, and a third dislocation has travelled out of the field of view in the upper right corner of the figure where the attending mesh refinement is clearly visible. To clarify the meaning of the contours in this figure, a close-up of dislocation $\mathrm{d} 2$ is shown in figure 8 . The plot accompanying this close-up is a plot of the edge component of interplanar slip, $\Delta$, due to the dislocation. The points labelled $A, B$, and $C$ indicate a point of no slip, a point near the centre of the dislocation core, and a point where slip of a full $\left(a_{0} / 6\right)\langle 112\rangle$ Burgers vector have taken place, respectively. Referring back to figure 7(b), we point out a final noteworthy feature of the deformation, namely the contours along the GB plane. These indicate that the atoms of the left-hand grain along the GB have rearranged to match the lattice of the grain on the right, resulting in a migration of the boundary.

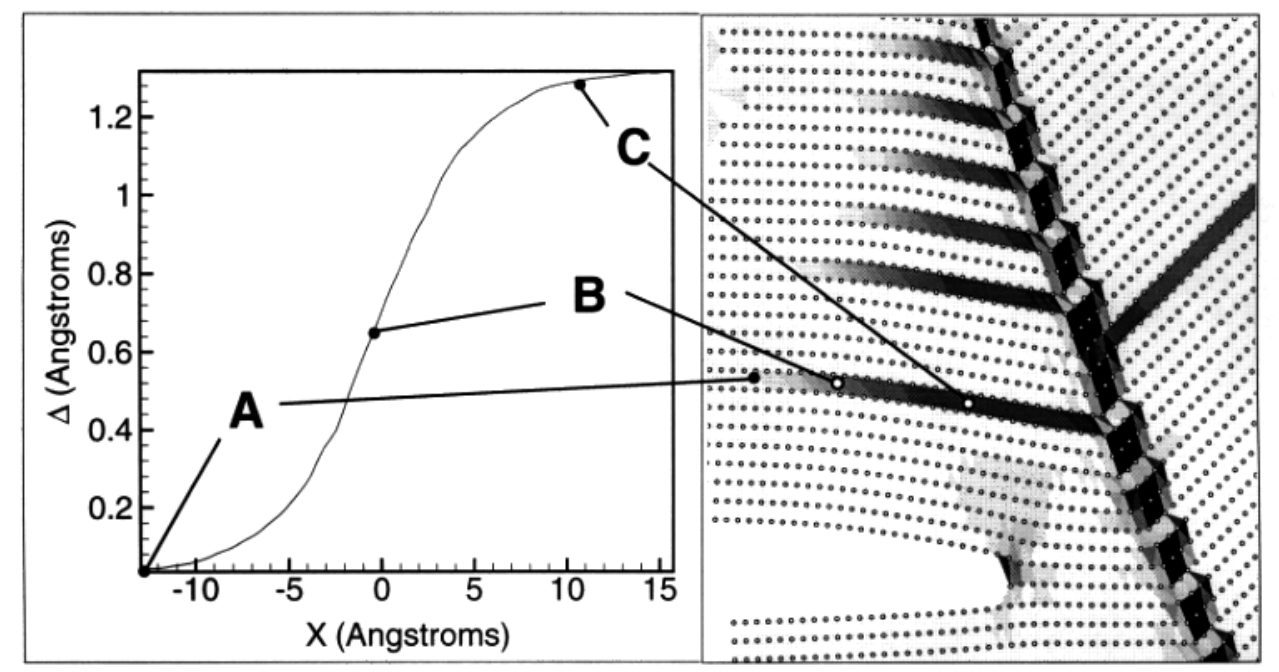

Figure 8. A close-up of the dislocation labelled $\mathrm{d} 2$ at time $t=3$. The contours between atoms correspond to the edge component on interplanar slip.

At $t=4$, the crack starts to propagate towards the GB by the separation of two (111) planes. This is made evident in figure 8(c), where the original crack-tip atom is denoted by ' $c t$ '. In addition, note that while the dislocation labelled $\mathrm{d} 1$ in the previous frame has travelled out of the field of view, d2 has actually been drawn back towards the GB. At this time, the transformation of the left-hand grain is substantial, so much so that the GB has bowed towards the crack tip. The patterned black and white region along the GB (the deformation causing this pattern will be discussed later) is the region that has transformed to accommodate the GB migration. Notice that this GB migration is not just an elastic straining of the grains, nor a rigid translation of the model, but a shuffling of atoms between crystal lattices. The atoms in the patterned region were originally part of the left grain, but by following the lines of the atomic planes, one can clearly see that they are now part of the grain on the right.

Two time steps later, at $t=6$, the crack has propagated up to the GB, and has been blunted due to the sliding of the left-hand grain up along the GB. Notice that due to the orientation of the GB with respect to the crack, this can only happen above the crack plane, and the result is a discontinuity in the GB at the crack. The new location of the GB can be 
seen by tracing atomic planes from one grain into the other. Note also that the dislocations immediately above the crack in the left grain have arranged themselves along a line just a short distance from the GB, while many of the dislocations that are further away (or that have travelled into the right-hand grain) continue to advance into the bulk crystal. While it cannot be seen in the field of view of this figure, the dislocation labelled d1 has travelled a distance of about $120 \AA$ at $t=6$, while the dislocations moving up and to the right have all travelled more than $200 \AA$ from the GB.

There are two aspects of this deformation process which we now consider in greater detail. The first is the emission of dislocations from the GB and into the right-hand grain, and the subsequent distance these dislocations travel. The second mechanism of interest is the migration of the GB due to the stress field around the crack tip.

The fact that GBs can act as a significant source of dislocations has been observed experimentally by several authors, as reviewed by $\mathrm{Li}$ (1981). Our simulation demonstrates that for this particular GB, the stresses due to the crack tip lead to a large number of dislocation emissions from the GB itself. The issue we discuss here is the distance which such dislocations travel, once they have been emitted into the bulk crystal. Recall that in order to follow a dislocation as it moves away from the GB, our model must continually refine. As mentioned earlier, this refinement was carried out only three times for every load step, and thus the final position of these long-range dislocations was around $200 \AA$ from the GB. Here, we try to estimate from linear elastic considerations the equilibrium distance that such a defect would travel. The development follows closely that of Ohr (1987) and others, in estimating the size of the 'dislocation free zone' ahead of a crack. The main difference in our model is the existence of a pinned dislocation in the GB itself, which is created at the same time as the mobile dislocation is emitted from the boundary. We begin constructing the situation of interest (a crack, GB, and dislocation) by superimposing the linear elasticity fields of defects. The GB itself can by built up as an array of dislocations, whose spacing is determined by the tilt angle of the GB. Note that such an array of dislocations has a highly localized stress field which dies off exponentially with distance into the neighbouring perfect crystals (Hirth and Lothe 1992). Thus, the crystals are essentially stress free and our dislocation will quickly cease to experience any force due to the GB itself. We are left then with the forces on the dislocation due to the crack and those due to a dislocation of equal and opposite Burgers vector held pinned in the GB itself. Finally, there are two forces at the atomic level, one due to the formation of a stacking fault as the partial dislocation moves and second, the Peierls force associated with moving a dislocation from one lattice position to the next. The picture, then, is as shown in figure 9 . We wish to determine the equilibrium position of the mobile dislocation, $r$, given a crack loaded with some stress intensity factor $K$ in mode I, a pinned dislocation at $d$, a stacking fault energy $\gamma$, and a Peierls stress $\sigma_{\mathrm{p}}$. The total force (per unit length) on the mobile dislocation is therefore (Lin and Thomson 1986)

$f=\frac{K b_{e}}{2 \sqrt{2 \pi r}} \sin \alpha \cos \frac{\alpha}{2}-\frac{\mu}{2 \pi}\left(b_{s}^{2}+\frac{b_{e}^{2}}{1-v}\right)\left[\frac{1}{2 r}+\frac{1}{r-d}-\frac{1}{r+d}\right]-\gamma-\sigma_{\mathrm{p}} b_{e}$.

The first term in this equation is the force on the dislocation due to the crack-tip stress field. The three terms in the square brackets are forces between dislocations, which includes a force due to the image of the mobile dislocation, a force due to the pinned dislocation, and a force between the image of the pinned dislocation and the mobile one. Finally, the last two terms represent the force due to the creation of a stacking fault and that due to the existence of a Peierls stress. There will be three distances $r$ for which this force will go to zero. Two of them will be within a few angstroms of the crack tip, and are in fact metastable equilibria. 
The third solution is a stable equilibrium at a relatively large distance from the crack tip. We have computed this distance for the load level associated with figure 7(b), using the following values of the equation parameters: $K=1.021 \mathrm{MPa} \mathrm{m}^{-1 / 2}, \mu=128.0 \mathrm{GPa}$, $v=0.33, \alpha=44.41^{\circ}, d=20 \AA,\left(b_{e}, b_{s}\right)=(1.2445,0.7185) \AA, \gamma=0.01450 \mathrm{~J} \mathrm{~m}^{-2}$ and $\sigma_{\mathrm{p}}=0.00068 \mu=0.0870 \mathrm{GPa}$. The value of the Peierls stress is taken from a calculation made by Shenoy and Phillips (1997) for a screw dislocation in aluminium. The resulting equilibrium distance is $r=3971 \AA$, which is significantly further than was observed in the simulation. It is perhaps not surprising that this distance agrees well with estimates for the size of the 'dislocation free zone' ahead of a crack, which is of the order of $5 \mu \mathrm{m}$ in fcc materials (Ohr 1985). The fact that our simulation led to much shorter distances leads us to conclude that there are important mesh effects entering our model. These effects will be difficult to circumvent, as following a dislocation $4000 \AA$ from the crack tip would lead to extremely large computational demands. As evidence that this mesh effect is not affecting the results in any significant qualitative way, we point out that this same simulation was run with mesh adaption deactivated. The emission of dislocations from the boundary, GB migration, load to crack propagation, and crack arrest by the GB were all observed to be approximately the same as what has been reported here. The difference being, of course, that no dislocations travelled further than the edges of the initial fully refined region of figure 6 . This suggests that even though these dislocations are being pinned by the mesh, they are not significantly affecting the subsequent deformation of the GB and crack.

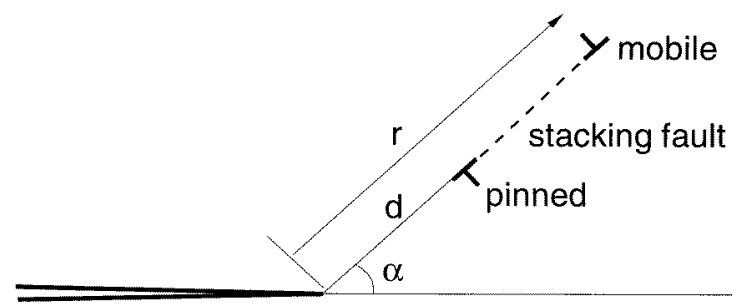

Figure 9. The model used to compute the equilibrium position $r$ of the mobile dislocation.

The second aspect of the simulation which we consider in greater detail is the migration of the boundary. A close-up of the crack-tip region from figure 7(c) is shown in figure 10 . Superimposed on this figure is a schematic representation of two rows of edge dislocations, with arrows joining the initial and final positions of each dislocation core. This is in fact the mechanism by which the boundary migrates, as evidenced by the dark slip traces beneath each arrow in the figure. This arrangement of dislocations is an array of undissociated $\langle 110\rangle$ edge dislocations on $\{111\}$ planes, and indeed it can be verified that these will lead to the correct tilt angle between the two grains. The motion of the boundary is then built up by the individual motions of the dislocations in the array. As the stress field around the crack tip builds, each dislocation experiences an increasing driving force. When the driving force on any one of the dislocations exceeds the lattice resistance, the dislocation moves. The nature of this driving force on the GB is the next topic of discussion.

The impetus for the migration of the GB is directly related to the jump in the elastic fields across the interface. This has been described in detail by Abeyaratne and Knowles (1990), Gurtin (1995), and others, and can be thought of in terms of a driving force on the boundary. This driving force is given by

$$
f=-[[\mathcal{E}]]+\left[\left[\boldsymbol{m} \boldsymbol{\sigma}^{\mathrm{T}} \boldsymbol{F} \boldsymbol{m}\right]\right]
$$




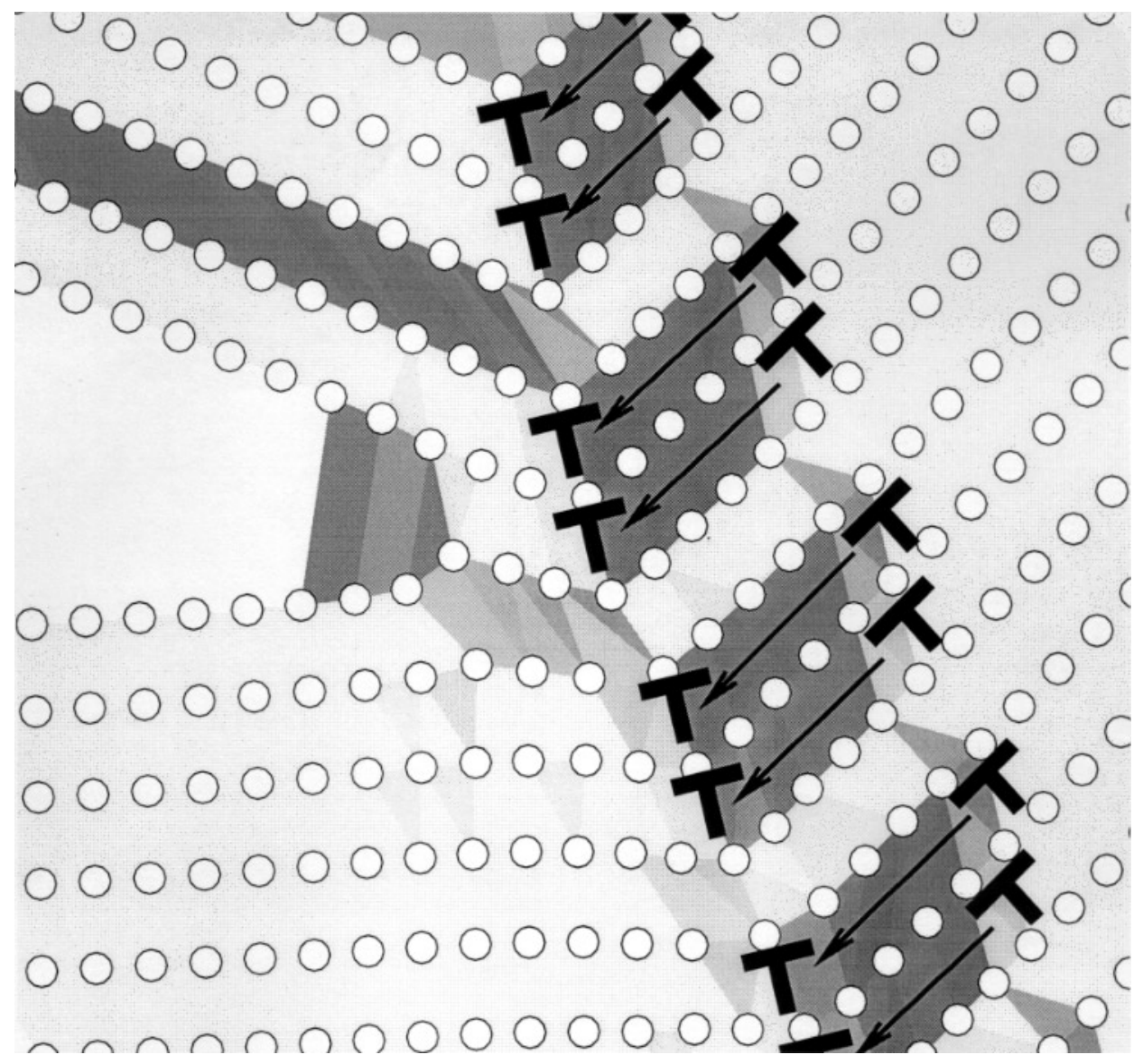

Figure 10. The mechanism of GB migration is the concerted slipping of the dislocations which comprise the boundary.

where $\mathcal{E}$ is the internal energy of the system, in this case given by the strain energy density, $\boldsymbol{m}$ is the normal to the boundary, and $[[\cdots]]$ represents the jump in a quantity across the interface. While it is not easy to compute the force directly from our QC analysis (recall that we do not rigorously have stress fields, only the atomic-level stresses) we can estimate the driving force based strictly on linear elastic considerations. In particular, if we consider the bicrystal as an elastic body with a jump in the moduli across the GB, it is possible to compute this driving force.

Within the finite-element method, it can be shown that the driving force of equation (14) can be re-written as a set of discrete forces applied to the nodes along the GB. These forces can be shown (Sussman and Bathe 1985) to be

$$
G_{\alpha}^{I}=-\int_{\Omega}\left(\mathcal{E} \frac{\partial N_{\alpha}}{\partial X^{I}}-P^{K J} F^{K I} \frac{\partial N_{\alpha}}{\partial X^{J}}\right) \mathrm{d} V
$$

where $G_{\alpha}^{I}$ is the force in the $I$ th direction applied to node $\alpha, \partial N_{\alpha} / \partial X^{I}$ is the gradient of the shape function at node $\alpha, P^{I J}$ is the first Piola-Kirchhoff stress, and $F^{I J}$ is the deformation gradient. By virtue of the compact support of the finite-element shape functions, the integral only involves the elements in contact with node $\alpha$, and can be evaluated using standard 
Gaussian quadrature methods. For the case of a linear elastic solid, this equation can be written in the form

$$
G_{\alpha}^{I}=-\int_{\Omega}\left(\frac{1}{2} \sigma^{P Q} \frac{\partial \psi^{P}}{\partial X^{Q}} \frac{\partial N_{\alpha}}{\partial X^{I}}-\sigma^{K J} \frac{\partial \psi^{K}}{\partial X^{I}} \frac{\partial N_{\alpha}}{\partial X^{J}}\right) \mathrm{d} V
$$

We have computed these nodal driving forces by performing an anisotropic, linear elastic, finite-element analysis. The mesh used was the same as in figure 6, except that midside nodes were added to each element and quadratic triangular elements were used for improved accuracy. The boundary nodes were held fixed to the same displacements as the boundary nodes in figure 7(a), and all interior nodes relaxed to the linear elastic solution. The nodal forces were then computed using (16), and interpolated along the entire GB using the finite-element shape functions. This driving force is plotted in figure 11(a) (normalized by $C_{11}=233.0 \mathrm{GPa}$ for nickel), against the distance along the GB (normalized by the lattice constant $a_{0}=3.52 \AA$ ). In figure $11(\mathrm{~b})$, the driving force is plotted directly on top of the finite-element mesh, scaled so that its maximum coincides with the maximum bow out of the boundary. Notice that this figure is plotted on the reference configuration of the finite-element mesh. The high-frequency noise in the force curve is a consequence of the finite-element mesh used to obtain the result, in conjunction with the need to interpolate nodal forces into a distributed force along the boundary. While any direct relation between the force on the boundary and the distance that it migrates must take into account the kinetics of boundary motion, some insight into these kinetics may be inferred by comparing the force distribution to the boundary profile. Above the plane of the crack, the fit between the driving force and the GB migration is quite good, while there is a large discrepancy below the crack plane. This difference in agreement seems to correlate with the fact that dislocation activity is mostly restricted to the segment of the GB above the crack plane. It is possible that the ability of a GB to emit dislocations is related to the GBs mobility. In the region above the crack tip, the GB was able to emit dislocations, which will presumably lower the driving force on the boundary and restrict the amount that the boundary can move. Below the crack plane, however, the inability of the GB to emit dislocations leads to a greater driving force and a subsequently greater GB migration. More study of this role of dislocation activity in the mobility of a GB is certainly warranted.

It is interesting to note the similarities between the GB migration observed in this simulation and that observed in the experimental studies of Würschum and Balluffi (1993) and Babcock and Balluffi (1989). While the nature of the driving force is very different in the two cases, the mechanisms of boundary migration seem closely related. Specifically, the experimental results for high-angle boundaries showed that boundary migration is achieved by the shuffling of atoms across the interface. Indeed, our calculations find such a mechanism, and elucidate the details of how this atomic shuffling takes place. The experiments report a 'jerky' boundary motion, indicative of a thermally activated mechanism. Our zero-temperature simulation demonstrates that the same mechanism can be initiated due to purely mechanical deformations. Finally, it is interesting that while the mechanism we observe is indeed a shuffling of atoms across the interface, the net result of the mechanism is the collective sliding of the dislocations comprising the boundary (cf figure 10). It may have been expected, due to the high angle of the boundary and high dislocation density, that the overlapping dislocation cores would invalidate this simple description of the boundary motion. However, in this case the highly planar structure of the dislocation cores enables them to exist in such close proximity without significant overlap. 

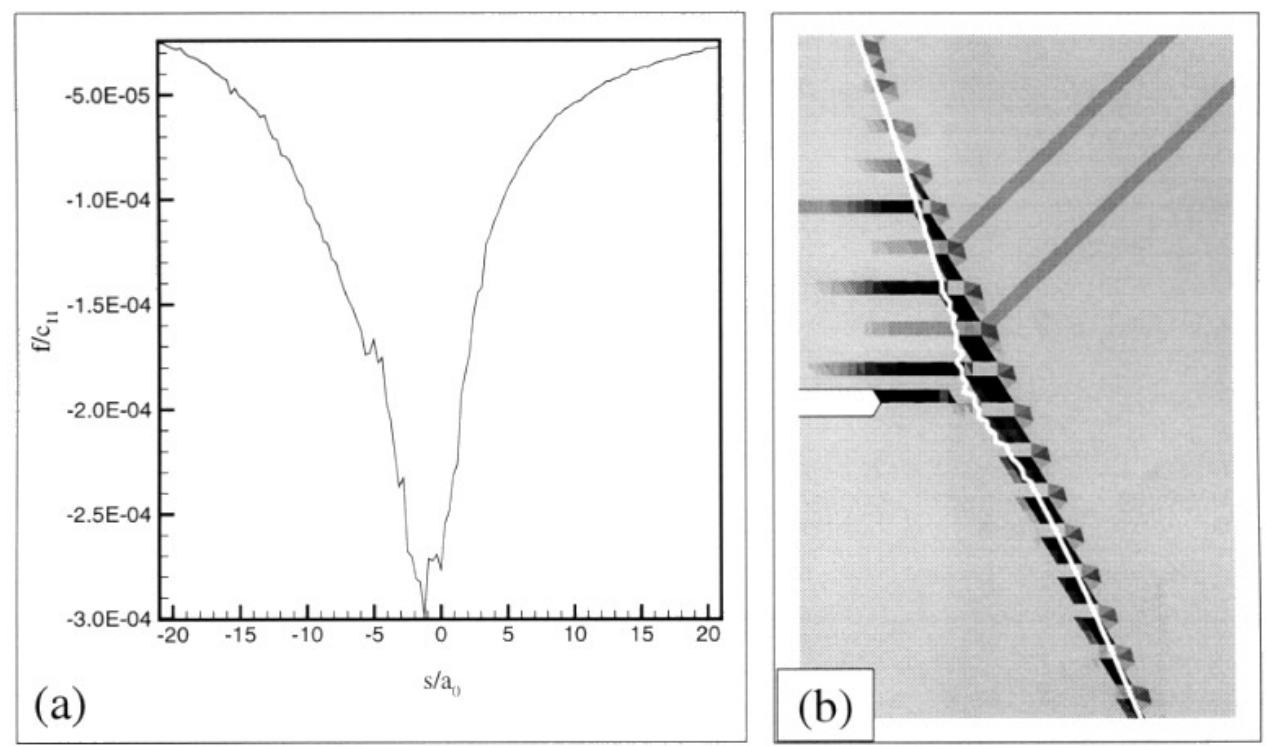

Figure 11. The linear elastic driving force on a $\Sigma=21(421)$ GB. (a) The normalized force per unit area as a function of the normalized distance $s / a_{0}$ along the boundary. ( $s=0$ is the point on the boundary directly in front of the crack tip.) (b) The force superimposed on the reference configuration at $t=4$ to compare the force to the amount of boundary migration. The patterned light/dark grey contours indicate the region through which the GB has migrated.

\subsection{Intergranular fracture}

As a second example, we study the interaction between a crack and the $\Sigma=5(\overline{1} 20)$ symmetric tilt boundary in nickel. The tilt axis of the boundary is in the [001] direction, and the tilt angle is $36.87^{\circ}$. The mesh design is very similar to that of the previous example, adjusted for the new crystallography, and the crack is initiated by removing a (110) plane from one of the crystals. The loading program is identical to that of the previous example. For comparison with the results of figure 7, the same load steps were used in this simulation, but a different selection of these load steps are shown in the results of figure 12. The differences in the behaviour are immediately apparent. Due to the lack of available slip systems in this 2D simulation of the new orientation, there is no dislocation activity. For this reason, we do not show the slip contours in this figure, and present only the atomic positions themselves around the crack tip. In addition, no mesh adaption was needed in this simulation as no dislocations form to carry the deformation beyond the initial fully refined region. The crack propagates to meet the boundary, but in this case the boundary does not migrate as in the previous example. While a driving force exists on this boundary as well, it is not high enough to lead to boundary motion. This can be thought of in terms of the dislocations which comprise the GB itself. In the previous example, the dislocations were mobile [110] dislocations on (111) planes. In this case, however, the GB is made up of sessile [100] dislocations on (010) planes. Such dislocations will require significantly higher driving forces to overcome lattice resistance. When the crack reaches the boundary it branches, running down along the boundary as well as up into the right grain, dragging the GB along and leaving in its wake a region where the right grain has transformed to match the crystallography of the grain on the left. 


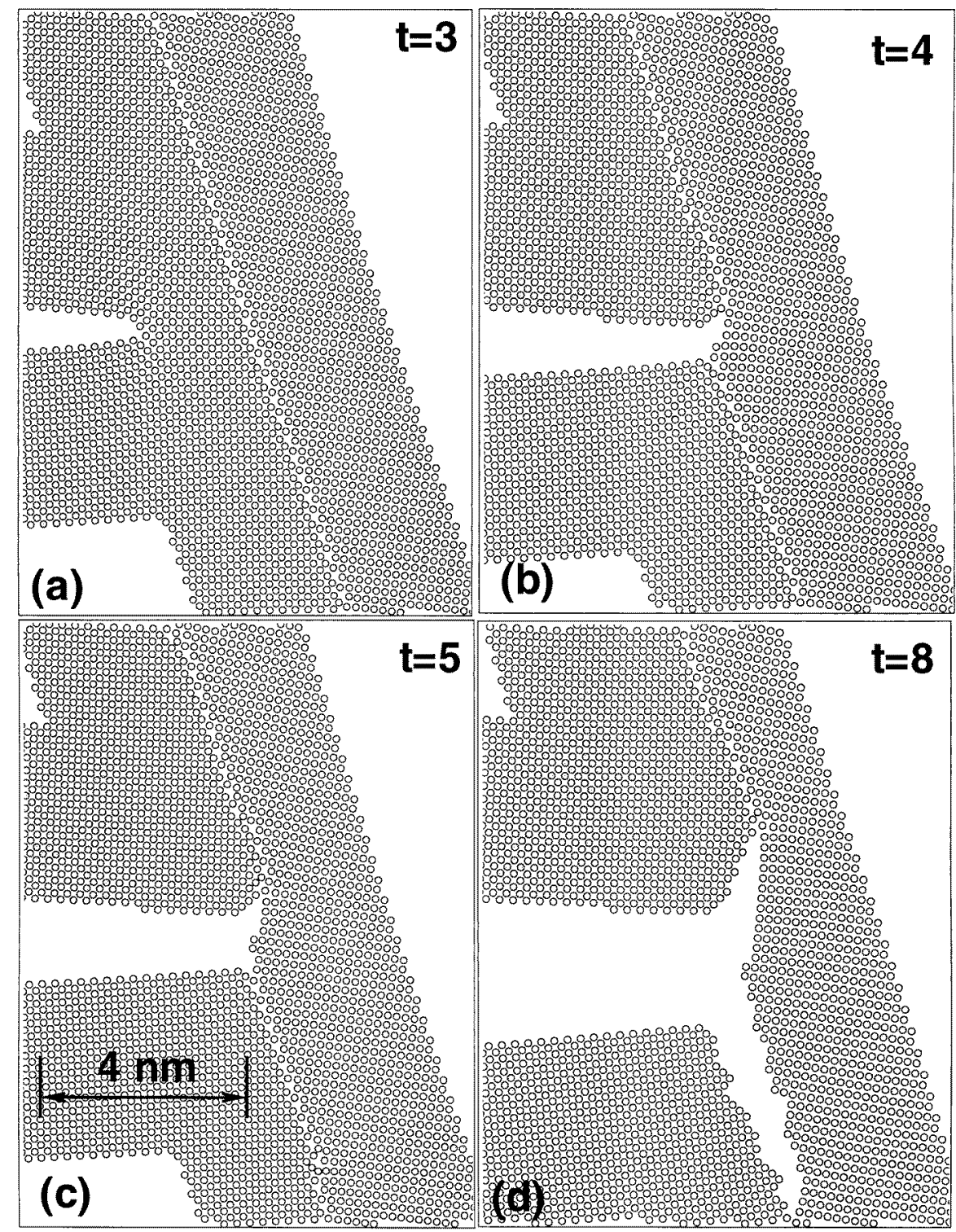

Figure 12. Simulation of a crack running up to a $\Sigma=5(\overline{1} 20)$ GB. See the text for details.

Intergranular fracture is not observed experimentally in fcc nickel (Briant 1990). The fact that we see this behaviour in our simulations may be explained on the basis of a number of approximations in our model. First, the fact that our model is not fully 3D can constrain certain deformation mechanisms that would be readily accessible to real materials. Second, our simulation is done at zero temperature, which makes the material more likely to behave in a brittle fashion. Finally, recall our earlier discussion of the EAM potentials used in these simulations. Rather than treating them literally as a description of nickel, we can think of them instead as representing a generic brittle fcc metal. In this case, we can compare our observations to the experimental studies of fracture in copper bicrystals 
carried out by Wang and Anderson (1991). In that work it was found that the symmetric tilt $\Sigma=5$ (130) boundary exhibited brittle fracture when a notch was introduced along the boundary plane. The boundary they studied is closely related to the one studied here, with the same $\Sigma$ number and tilt axis, but a different boundary plane.

While it may be difficult to make direct contact between this simulation and experimental observations, the differences between figures 7 and 12 demonstrate the ability of the QC model to capture a wide range of material response. Essentially, there is only one difference between the input to the simulation of figure 7 and that of figure 12-a change in the orientation of the two crystals.

\section{Conclusion}

We have made use of the QC method to study fracture in single crystals and in the presence of GBs. In single crystals, the results were used to quantify the error associated with approximate models of crack-tip plasticity like that proposed by Rice (1992). Two crack orientations were considered, one which failed in what is commonly referred to as a brittle mode - the advance of the crack by cleavage between atomic planes. In the second orientation, the crack tip emitted a dislocation, which we refer to as a ductile response.

It was found that in the brittle orientation, the Griffith analysis is in good agreement with the atomistic results, although the effect of atomic scale crack blunting leads to a small but significant increase in the critical energy release rate. On the other hand, the Rice criterion used to discern between brittle or ductile behaviour was qualitatively incorrect in this case, predicting dislocation emission for the brittle crack orientation.

In the case where the crack did not advance, but failed by the emission of dislocations, the Rice model underpredicted the critical load to failure by about $45 \%$. We noted that a number of authors have presented corrections to the Rice approach whose effect is to increase the predicted critical load for dislocation emission. Our results suggest that this is the correct sign on the correction, but it is unclear whether these new models capture all of the relevant physics of the problem. In an effort to understand the reason for the failure of the Rice analysis, we examined the extent of the nonlinear region in the vicinity of the crack tip. We have concluded that nonlinearity, while indeed confined to a region only a few lattice spacings away from the crack tip, is not confined to a single atomic plane, as assumed in the cohesive zone models. This suggests that a model which can extend the nonlinear region beyond a single plane may be a more faithful representation of the crack-tip deformation. Finally, a comparison between the atomic scale stresses near the crack tip and simple estimates of the theoretical strength based on the UBER and $\gamma$-surface was made. The results show that for the cases considered, the atomic-level stresses attain significantly higher values than the simple estimates suggest.

In studying the interaction between cracks and GBs, we have revealed a number of interesting deformation mechanisms. In the example of the $\Sigma=21$ (421) boundary, the approaching crack induces the GB to migrate towards the crack tip, at the same time activating a number of dislocation sources along the boundary. As a result, the advance of the crack is accompanied by a significant amount of plastic flow originating from the GB. This suggests that grain boundaries in fcc metals may be significant sources of dislocations, and an important contribution to the hardening behaviour of such materials. Finally, the advance of the crack is arrested as it is blunted by the boundary, a process that is accommodated by GB sliding.

For the $\Sigma=5(\overline{120)}$ ) boundary, the lack of any available slip systems (due in part to the reduced dimensionality of our model) leads to intergranular fracture. The crack reaches the 
boundary and then branches, moving along the boundary in one direction and up into the neighbouring grain in another direction.

In both of the GB simulations, the observed behaviour was rationalized based on continuum mechanics arguments. The migration of the $\Sigma=21(421)$ boundary was correlated to a configurational force on the GB due to the change in the elastic moduli tensor across the interface. The mechanism of the boundary motion could be further understood as the collective motion of the individual dislocations of which the boundary is comprised. On the other hand, the observation that the $\Sigma=5(120)$ boundary did not migrate can be understood on the basis of the sessile nature of its constituent dislocation cores.

There are still many questions to be answered regarding fracture at the atomic scale. One general conclusion of this work is that modelling must truly address the issue of 3D configurations in order to make proper contact with experiments. Simulations with reduced dimensionality tend to freeze out possible deformation paths, such as dislocation emission along planes that are oblique to both the crack plane and the crack front (Xu et al 1995). In addition, the need for a more general analytical framework to describe fracture at the atomic scale must be developed. While simulations of fracture processes are valuable tools for exploring possible fracture processes, it is through an analytical framework that the greatest potential for insight and prediction is gained.

\section{Acknowledgments}

We are grateful to C Briant, A Carlsson, R Clifton, B Freund, J R Rice, D Rodney, J Schiotz, and V B Shenoy for discussions, to S W Sloan for use of his Delaunay triangulation code and to M Daw and S Foiles for the use of their Dynamo code. We are also grateful to AFOSR who supported this work under grant No F49620-95-I-0264 and the NSF through grants CMS-9414648 and the Brown MRSEC DMR-9632524, the DOE through grant DE-FG02-95ER14561. RM thanks the NSERC for its support.

\section{References}

Abeyaratne R and Knowles J K 1990 On the driving traction acting on a surface of strain discontinuity in a continuum J. Mech. Phys. Sol. 38 345-60

Abraham F F, Schneider D, Land B, Lifka D, Skovira J, Gerner J and Rosenkrantz M 1998 Instability dynamics in three-dimensional fracture: an atomistic simulation J. Mech. Phys. Sol. 45 1461-71

Armstrong R 1966 Cleavege crack propagation within crystals by the Griffith mechanism versus a dislocation mechanism Mater. Sci. Eng. 1 251-4

Babcock S E and Balluffi R W 1989 Grain boundary kinetics-II. In situ observations of the role of grain boundary dislocations in high-angle boundary migration Acta Metall. 37 2367-76

Briant C L 1990 On the chemistry of grain boundary segregation and grain boundary fracture Metall. Trans. A 21 2339-53

Cheung K S and Yip S 1990 Brittle-Ductile transition in intrinsic fracture behavior of crystals Phys. Rev. Lett. 65 2804-7

-1991 Atomic-level stress in an inhomogeneous system J. Appl. Phys. 70 5688-90

Cleri F, Phillpot S R and Wolf D 1998a Comparison between atomistic and continuum mechanics modelling of grain-boundary fracture Mater. Res. Soc. Symp. Proc to appear

Cleri F, Phillpot S R, Wolf D and Yip S 1998b Atomistic simulations of materials fracture and the link between atomic and continuum length scales J. Am. Ceram. Soc. 81501

Daw M S, Baskes M I, Bisson C L and Wolfer W G 1986 Application of the embedded atom method to fracture, dislocation dynamics and hydrogen embrittlement Modeling Environmental Effects on Crack Growth Processes (Warrendale, PA: Metallurgical Society) pp 99-124

DeLorenzi H G 1982 On the energy release rate and the J-integral for 3-D crack configurations Int. J. Fracture 19 183-93 
Egami T and Srolovitz D 1982 Local structure fluctuations in amorphous and liquid metals: a simple theory of the glass transition J. Phys. F: Met. Phys. 12 2141-63

Foiles S M, Baskes M I and Saw M S 1986 Embedded-atom-method functions for the fcc metals $\mathrm{Cu}, \mathrm{Ag}, \mathrm{Au}, \mathrm{Ni}$, Pd, Pt and their alloys Phys. Rev. B 33 7983-91

Freund L B 1990 Dynamic Fracture Mechanics (Cambridge: Cambridge University Press)

Gumbsch P 1995 An atomistic study of brittle fracture: toward explicit failure criteria from atomistic modeling J. Mater. Res. 10 2897-907

Gumbsch P and Beltz G E 1995 On the continuum versus atomistic descriptions of dislocation nucleation and cleavage in nickel Modelling Simulation Mater. Sci. Eng. 3 597-613

Gurtin M E 1995 The nature of configurational forces Arch. Rational Mech. Anal. 131 67-100

Hirth J P and Lothe J 1992 Theory of Dislocations (Malabar, FL: Krieger)

Holian B L and Ravelo R 1995 Fracture simulations using large-scale molecular dynamics Phys. Rev. B 51 $11275-88$

Juan Y-M, Sun Y and Kaxiras E 1996 Ledge effects on dislocation emission from a crack tip: a first-principles study for silicon Phil. Mag. Lett. 73 233-40

Kelly A, Tyson W and Cottrell A 1967 Ductile and brittle crystals Phil. Mag. 15 567-85

Kohlhoff S, Gumbsch P and Fischmeister H F 1991 Crack propagation in bcc crystals studied with a combined finite-element and atomistic model Phil. Mag. A 64 851-78

Lawn B 1993 Fracture of Brittle Solids (Cambridge: Cambridge University Press)

Li J C M 1981 Dislocation sources Dislocation Modelling of Physical Systems (Oxford: Pergamon) pp 498-518

Lin L-H and Thomson R 1986 Cleavage, dislocation emission and shielding for cracks under general loading Acta Metall. 34 187-206

Lomdahl P S, Beazley D M, Tamayo P and Gronbech-Jensen N 1993 Multi-million particle molecular dynamics on the CM-5 Int. J. Mod. Phys. C 4 1075-84

Miller R, Ortiz M, Phillips R, Shenoy V and Tadmor E 1998 Quasicontinuum models of fracture and plasticity Eng. Fracture Mech. at press

Ohr S M 1985 An electron microscope study of crack tip deformation and its impact on the dislocation theory of fracture Mater. Sci. Eng. 72 1-35

-1987 Dislocation-crack interaction J. Phys. Chem. Sol. 48 1007-14

Paskin A, Massoumzadeh B, Shkula K, Sieradzki K and Dienes G J 1985 Effect of atomic crack tip geometry on local stresses Acta Metall. 33 1987-96

Press W H, Teukolsky S A, Vetterling W T and Flannery B P 1992 Numerical Recipes in FORTRAN: The Art of Scientific Computing 2nd edn (Cambridge: Cambridge University Press)

Rice J R 1968 A path independent integral and the approximate analysis of strain concentration by notches and cracks J. Appl. Mech. 35 379-86

1992 Dislocation nucleation from a crack tip: an analysis based on the Peierls concept J. Mech. Phys. Sol. 40 239

Rice J R and Thomson R 1974 Ductile versus brittle behaviour of crystals Phil. Mag. 29 73-97

Rodney D and Phillips R 1998 Phys. Rev. Lett. submitted

Rose J H, Ferrante J and Smith J R 1981 Universal binding energy curves for metals and bimettalic interfaces Phys. Rev. Lett. 47 675-8

Schiotz J, Canel L M and Carlsson A E 1997 Effects of crack tip geometry on dislocation emission and cleavage: a possible path to enhanced ductility Phys. Rev. B 55 6211-21

Sharon E, Gross S P and Fineberg J 1996 Energy dissipation in dynamic fracture Phys. Rev. Lett. 76 2117-20

Shen Z, Wagoner R H and Clark W A T 1988 Dislocation and grain boundary interactions in metals Acta Metall. 36 3231-42

Shenoy V B and Phillips R 1997 Finite-sized atomistic simulations of screw dislocations Phil. Mag. A 76 367-85

Shenoy V B, Miller R, Rodney D, Tadmor E B, Phillips R and Ortiz M 1998 An adaptive finite element approach to atomic scale mechanics: the quasicontinuum method J. Mech. Phys. Sol. at press

Shih C F and Moran B 1987 Crack tip associated domain integrals from momentum and energy balance Eng. Fracture Mech. 27 615-42

Sih G C and Liebowitz H 1968 Mathematical theories of brittle fracture Fracture: An Advanced Treatise 2 108-29

Sinclair J E and Lawn B R 1972 An atomistic study of cracks in diamond-structure crystals Proc. R. Soc. Lond. A 32983

Sun Y, Beltz G E and Rice J R 1993 Estimates from atomic models of tension-shear coupling in dislocation nucleation from a crack tip Mater. Sci. Eng. A $17067-85$

Sussman T and Bathe K-J 1985 The gradient of the finite element variational indicator with respect to nodal point coordinates: an explicit calculation and applications in fracture mechanics and mesh optimization Int. J. Num. 
Meth. Eng. 21 763-74

Tadmor E B, Ortiz M and Phillips R 1996 Quasicontinuum analysis of defects in solids Phil. Mag. A 73 1529-63

Thomson R and Carlsson A E 1994 Intrinsic ductility criterion for materials Phil. Mag. A 70 893-903

Thomson R M 1973 The fracture crack as an imperfection in a nearly perfect solid Ann. Rev. Mater. Sci. 3 31-51

Vitek V 1968 Intrinsic stacking faults in body-centered cubic crystals Phil. Mag. 18 773-86

Vitek V and Egami T 1987 Atomic level stresses in solids and liquids Phys. Status Solidi b 144 145-56

Wang J-S and Anderson P M 1991 Fracture behavior of embrittled fcc metal bicrystals Acta Metall. 39 779-92

Würschum R and Balluffi R W 1993 In-situ study of the migration of grain boundary facets in Au bicrystals under high driving forces Phys. Status Solidi a 136 323-36

Xu X-P, Argon A S and Ortiz M 1995 Nucleation of dislocations from crack tips under mixed modes of loading: implications for brittle versus ductile behaviour of crystals Phil. Mag. A 72415

Zhou S J, Beazley D M and Lomdahl P S 1997 Large-scale molecular-dynamics simulations of three-dimensional ductile fracture Phys. Rev. Lett. 78 479-82

Zhou S J, Carlsson A E and Thomson R 1993 Dislocation nucleation and crack stability: lattice Green's-function treatment of cracks in a model hexagonal lattice Phys. Rev. B 47 7710-18

1994 Crack blunting effects on dislocation emission from cracks Phys. Rev. B 72 852-5 